\title{
Grammatical Gender and Anthropomorphism: \\ "It" Depends on the Language
}

\author{
ALICAN MECIT \\ HEC Paris \\ TINA M. LOWREY \\ HEC Paris \\ L. J. SHRUM \\ HEC Paris
}

\begin{abstract}
AUTHOR NOTES
Alican Mecit, Department of Marketing, HEC Paris, alican.mecit@hec.edu. Tina M. Lowrey, Department of Marketing, HEC Paris, lowrey@hec.fr. L. J. Shrum, Department of Marketing, HEC Paris, shrum@hec.fr. This research was supported by a research grant from the HEC Foundation of HEC Paris and Investissements d'Avenir (ANR-11-IDEX-0003/Labex Ecodec/ANR-11-LABX-0047) awarded to the second and third authors. This article is based on a portion of the doctoral dissertation of the first author under the co-supervision of the second and third authors. Supplementary materials are included in the web appendix accompanying the online version of this article. The data for some studies were collected at the INSEAD-Sorbonne University Behavioral Lab.
\end{abstract}




\title{
CONTRIBUTION STATEMENT
}

Consumer research on anthropomorphism has identified a number of effects of anthropomorphism on consumer judgments in a variety of contexts. However, the antecedents of anthropomorphism have received relatively little attention. In this research, we identify a novel antecedent of anthropomorphism: language. Across 10 studies, we demonstrate that the grammatical structure of language-in particular, whether languages grammatically distinguish between humans and non-humans-influences anthropomorphism tendencies. We show that speakers of languages that do not distinguish between humans and non-humans (it-less languages) anthropomorphize more than do speakers of languages that do make this distinction (non-it-less languages).

Our research makes three major contributions. First, we contribute to the literature on consumer anthropomorphism by identifying the grammatical gender system of a language as a novel antecedent of anthropomorphism. To our knowledge, this is the first research to investigate linguistic antecedents of anthropomorphism. Second, we contribute to the broader debate on the extent to which language influences thought. We show that the grammatical features of language can affect higher-level cognitive mechanisms such as attitudes, purchase intentions, and choice, thus, our results provide empirical evidence in favor of the linguistic relativity thesis. Third, we contribute to marketing practice by showing that managers can leverage nonconscious "grammatical anthropomorphism" to influence consumer perceptions, attitudes, and behavior.

\begin{abstract}
Consumers often anthropomorphize non-human entities. In this research, we investigate a novel antecedent of anthropomorphism: language. Some languages (e.g., English) make a grammatical distinction between humans (he, she) and non-humans (it), whereas other languages (e.g., French) do not (all objects are gender-marked). We propose that such grammatical structures of languages influence the way individuals mentally represent non-human entities, and as a result, their generalized tendencies to anthropomorphize such entities. Across 10 studies, we provide evidence that speakers of languages that do not grammatically distinguish between humans and non-humans (it-less languages) anthropomorphize more than do speakers of languages that do make this distinction (non-it-less languages). We demonstrate the effects across natural languages (French, Turkish, English) and by manipulating grammatical gender. We show that the effects are observable in naturally occurring consumer contexts (e.g., secondary sales data), and that gender-marking in it-less languages influences consumers' interactions with brands, even though the gender-markings are semantically arbitrary, and that these effects occur nonconsciously. Our findings have implications for the broader debate on the extent to which languages influences thought, and also suggest ways in which managers can leverage nonconscious grammatical anthropomorphism to influence consumer perceptions, attitudes, and behavior.
\end{abstract}

Keywords: Anthropomorphism, linguistics, categorization. 
Consumers' interactions with brands and products occupy a central part of their lives. The nature of these interactions, however, varies greatly. On the one hand, consumers buy, use, and eventually dispose of products without giving much thought to their relations with and feelings about those products. On the other hand, consumers talk to their belongings, name them, and even consider them as members of their families (Aggarwal and McGill 2007; Epp and Price 2009). Although these consumption objects are of course not human, consumers can come to treat them as if they are. This tendency to ascribe human characteristics to non-human entities, or anthropomorphism, has been a frequent topic of consumer research in recent years (Yang, Aggarwal, and McGill 2020). The general finding is that anthropomorphizing products or brands has a number of positive effects on marketing outcomes, and consequently marketers encourage anthropomorphism of their products in a variety of ways (e.g., product design, promotions, spokes-characters, etc.).

Although considerable research has investigated the consequences of anthropomorphism and identified sizable effects on consumer behavior (Hur, Koo, and Hofmann 2015; Kim, Peng, Chen and Zhang 2016; Mourey, Olson, and Yoon 2017), the antecedents of anthropomorphism have received relatively less attention. In this research, we take up the question of what makes some consumers more likely to attribute human characteristics to non-humans. Anthropomorphic thinking is considered by most to be both natural and universal (Dacey 2017; Harris and Fiske 2008; Hume 1957). Nevertheless, both individuals and cultures differ on the extent to which they spontaneously anthropomorphize (Waytz, Cacioppo, and Epley 2010). Some cultures are known for their anthropomorphic worldviews, such as the Shinto belief in Japanese culture, which views mountains, lakes, and rivers as having sacred spirits (Teeuwen and Scheid 2002), or the Native 
American folklore whose tales feature various anthropomorphic animals (e.g. coyote; Alexander 2012).

In this research, we propose a novel antecedent of anthropomorphism: language. Specifically, we test the proposition that certain grammatical structures of languages influence the way individuals mentally represent non-human entities, and as a result, their generalized tendencies to anthropomorphize such entities. One way in which languages differ in the way they treat non-humans is their grammatical gender systems. For example, some languages, such as French, assign gender to all nouns, including non-humans, through definite $(l a, l e)$ and indefinite (une, un) articles, whereas other languages, such as English, do not (the and $a$ are genderless). Similarly, languages differ on the extent to which they have separate pronouns for human and non-human entities. English makes this distinction with the pronoun it, which is used to refer to non-human nouns. In contrast, as with articles, French does not have a separate pronoun for nonhuman entities, but instead assigns gendered pronouns for all entities. Therefore, when referring to non-human entities, French speakers use either il (he) or elle (she), depending on the grammatical gender of the object. For example, when referring to a red table, in English it would be "it is red," whereas for French speakers, it would be "elle est rouge" (she is red).

Language shapes thought along many dimensions (von Humboldt and von Humboldt 1999; Lucy 1997; Whorf 1952), and we propose that one dimension is anthropomorphic thought. The language people use when they talk about a non-human entity exerts a significant influence on their tendency to anthropomorphize the non-human entity. For example, when people use pronouns referring to humans, such as he or she, to refer to a non-human entity, they become more likely to regard the non-human entity in human terms (Aggarwal and McGill 2007). Because one's native language draws the limits on the way one speaks about non-human entities, 
and in some languages (e.g., French and Turkish) it is simply not possible to talk about nonhumans without using pronouns referring to humans, we propose that whether languages assign gender to all nouns, or whether they have different pronouns for humans and non-humans, affects speakers' tendencies to anthropomorphize, which in turn influences the way people interact with non-human entities, particularly consumer products.

Thus, we propose that speakers of languages that do not grammatically distinguish between human and non-human entities will anthropomorphize more than speakers of languages that do make this distinction. More specifically, we propose that grammatical gender induces a process of grammatical anthropomorphism in which linguistic gender markers orient attention toward similarities or differences between human and non-human entities, depending on properties of the languages' grammatical gender system. This proposition also resonates with the cognitive theory of language use, which posits that pronoun systems in a language can affect the structure of relations between different entities and contribute to the construction of social reality (Langacker 1987; Logan 1986; Mühlhäusler and Harré 1990).

We test this proposition in 10 studies that provide support for the hypotheses both in studies that compare anthropomorphism as a function of natural differences in languages (e.g., French, English, Turkish) and manipulate the grammatical distinction between human and nonhuman nouns within language. We show that the effects generalize across multiple measures of anthropomorphism. We further show that the effects are observable in naturally occurring consumer contexts (e.g., secondary sales data), and that the language effects have downstream effects on consumer attitudes, purchase intentions, and consequential choice.

Our research integrates insights in linguistics and anthropomorphism, and makes several contributions to the consumer literature. First, we identify a novel antecedent of 
anthropomorphism, language, showing that the absence (vs. presence) of grammatical distinction between human and non-human nouns in a language predisposes individuals to anthropomorphism. To the best of our knowledge, this is the first paper to investigate the linguistic antecedents of anthropomorphism. Second, and related, we contribute to the literature on consumer anthropomorphism. We show that in some cultures, anthropomorphism occurs spontaneously and nonconsciously through the language structure, and explicit cues that are reminiscent of human form are not necessary-and can even be counterproductive-for anthropomorphic thought. Third, we contribute to the literature on linguistic determinants of thought and behavior, and the larger theoretical debate on the extent to which language influences thought. By showing that the grammatical features of language can affect higher-level cognitive mechanisms such as attitudes, purchase intentions, and choice, our results provide empirical evidence in favor of the linguistic relativity thesis. Finally, from an applied perspective, marketers can systematically take into account the effect of linguistic cues and predict the likely effects on consumer behavior.

\section{ANTHROPOMORPHISM}

Marketers have long been aware of the benefits of product and brand anthropomorphism, which they have encouraged in a number of ways. The most obvious one is through spokescharacters like the Duracell Bunny, Michelin Man, and the Laughing Cow (La Vache qui rit). Anthropomorphism can also be induced through product design (e.g., torso-shaped perfume bottle of Le Male Jean-Paul Gaultier and the humanlike face of the LG Hub Robot). Numerous studies have provided empirical evidence of the benefits of consumer anthropomorphism (Yang 
et al. 2020). Inducing anthropomorphism by altering the appearance of a product (e.g., a smiling car grille or hands of a clock) increases product liking (Aggarwal and McGill 2007; Labroo, Dhar, and Schwarz 2008). Priming anthropomorphic cues increases consumers' intentions to hold on to (rather than replace) their current products (Chandler and Schwarz 2010) and their intentions to contribute to prosocial causes like environmental conservation (Ahn, Kim, and Aggarwal 2014; Tam, Lee, and Chao 2013). Although the mechanisms for each effect may differ slightly, and there are potential downsides to product anthropomorphism, the general mechanism is that encouraging the view of products as human allows for deeper connections between consumers and their products, which has positive effects on marketing outcomes (for a review, see Yang et al. 2020).

Antecedents of Anthropomorphism

Although a number of frameworks for why people anthropomorphize have been offered (cf. Guthrie 1993; Yang et al. 2020), for our purposes, we focus on the three-factor model articulated by Epley, Waytz, and Cacioppo (2007). They propose three determinants of anthropomorphism, two of which are motivational, and the other one cognitive. One motivation for anthropomorphism is social connection. Imbuing products with (positive) human traits allows consumers to feel closer to their products and to interact with them as if they were human. Thus, people who are lonely are more likely than nonlonely people to anthropomorphize things like technological gadgets (Epley et al. 2008), and interacting with anthropomorphized products can mitigate negative effects of loneliness and social exclusion and increase vitality (cf. Chen, Sengupta, and Adaval 2018; Chen, Wan, and Levy, 2017; Mourey, Olson, and Yoon 2017). 
A second motivational determinant of anthropomorphism is effectance motivation, which is the desire to understand, explain, and control behavior of other agents. Perceiving non-human entities to have minds like humans helps people make sense out of unpredictable situations or products, and provides feelings of increased control because people tend to make dispositional attributions to explain others' behavior. For example, in one study, participants were more likely to anthropomorphize unpredictable gadgets than predictable ones (Waytz et al. 2010), and in another study, judged an autonomous vehicle as more likely to perform effectively the more it acquired anthropomorphic features (Waytz, Heafner, and Epley 2014).

The third antecedent of anthropomorphism proposed by Epley et al. $(2007,886)$ is what they term "elicited agent knowledge," which refers to the accessibility of anthropomorphic knowledge structures. These knowledge structures can be elicited situationally, for example through explicit cues. Reminding consumers of the human form increases the temporary accessibility of knowledge about humans when interacting with objects (Waytz, Cacioppo, and Epley 2010). Consumers are particularly likely to treat objects as humans when the objects have humanlike features (Haley and Fessler 2005; Jipson and Gelman 2007; Woodward 1999), and as noted earlier, product appearance can be easily manipulated to induce anthropomorphism (Aggarwal and McGill 2007).

The accessibility of anthropomorphic knowledge structures also differs across people. That is, although anthropomorphism may be a universal tendency, not everyone does it to the same degree. Individual differences in the extent to which one attributes human characteristics to non-humans are stable over time, and may arise for a number of reasons, including differences in culture, norms, education, experience, and cognitive reasoning styles, among others (Waytz et al. 2010). Culture, in particular, may influence anthropomorphic tendencies by providing distinct 
norms and ideologies about how people relate to other humans, and also to the natural world, and a number of studies have documented cultural differences in patterns and types of anthropomorphism (Ross et al. 2003; for a review, see Epley et al. 2007).

We propose that one culture-related determinant of anthropomorphic tendencies is language. In particular, we propose that systematic differences in the way languages treat humans and non-humans influence the accessibility and content of human knowledge structures when reasoning about non-humans, which in turn influence anthropomorphism tendencies. In the next section, we provide a theoretical framework for this prediction.

\section{LANGUAGE, THOUGHT, AND BEHAVIOR}

The proposition that language shapes thought (and thus subsequent behavior), often called the Sapir-Whorf hypothesis of linguistic relativity, argues that language shapes the way people perceive and understand the world (Whorf 1952), and consequently people who speak different languages perceive the world differently (Logan 1986). Although the notion of linguistic relativity has its critics (e.g., Pinker 1994), relatively recent findings strongly support the proposition, showing interactive relations between language and cognitive functions in several domains, including color perception (Roberson, Pak and Hanley 2008), time perception (Casasanto and Boroditsky 2008), emotions (Gendron, et al. 2012), and motion (Meteyard, Bahrami and Vigliocco 2007). For example, English and Korean languages differ on the information their verbs specify when describing motion events. English tends to specify the manner of motion (e.g., flying, running), whereas Korean tends to omit manner information. These differences affect memory for details of events, with English speakers remembering more 
manner-relevant details than Korean speakers (Oh, 2003; reported in Casasanto 2016). Thus, language presumably affected the encoding and retrieval of situation-relevant knowledge structures.

Of particular interest to our research, pronouns in a language can affect the structure of relations between different entities and contribute to the construction of social reality (Langacker 1991; Mühlhäusler and Harré 1990). For example, the use of subject pronouns is obligatory in some languages, such as English and French, whereas other languages do not require the expression of such pronouns (e.g., Spanish). Research shows that this practice of pronoun drop in a language is correlated with lower levels of individualism (Kashima and Kashima 1998).

\section{Grammatical Gender Systems}

One way in which languages differ is how their grammatical systems assign gender (see figure 1). Some languages, such as French and Spanish, assign gender to all nouns through definite articles and gendered pronouns, and are referred to as gendered languages. Other languages, such as English, typically do not assign gender to all nouns, but mark gender through pronouns for humans (he and she). For non-humans, the pronoun is non-gendered (it); such languages are referred to as natural gender languages. A third category is genderless languages, such as Turkish, in which gender is not marked for either humans or non-humans. In these languages, a single pronoun is used to refer to all entities (e.g., $o$ in Turkish).

\section{FIGURE 1}

GRAMMATICAL GENDER SYSTEMS IN DIFFERENT LANGUAGES 


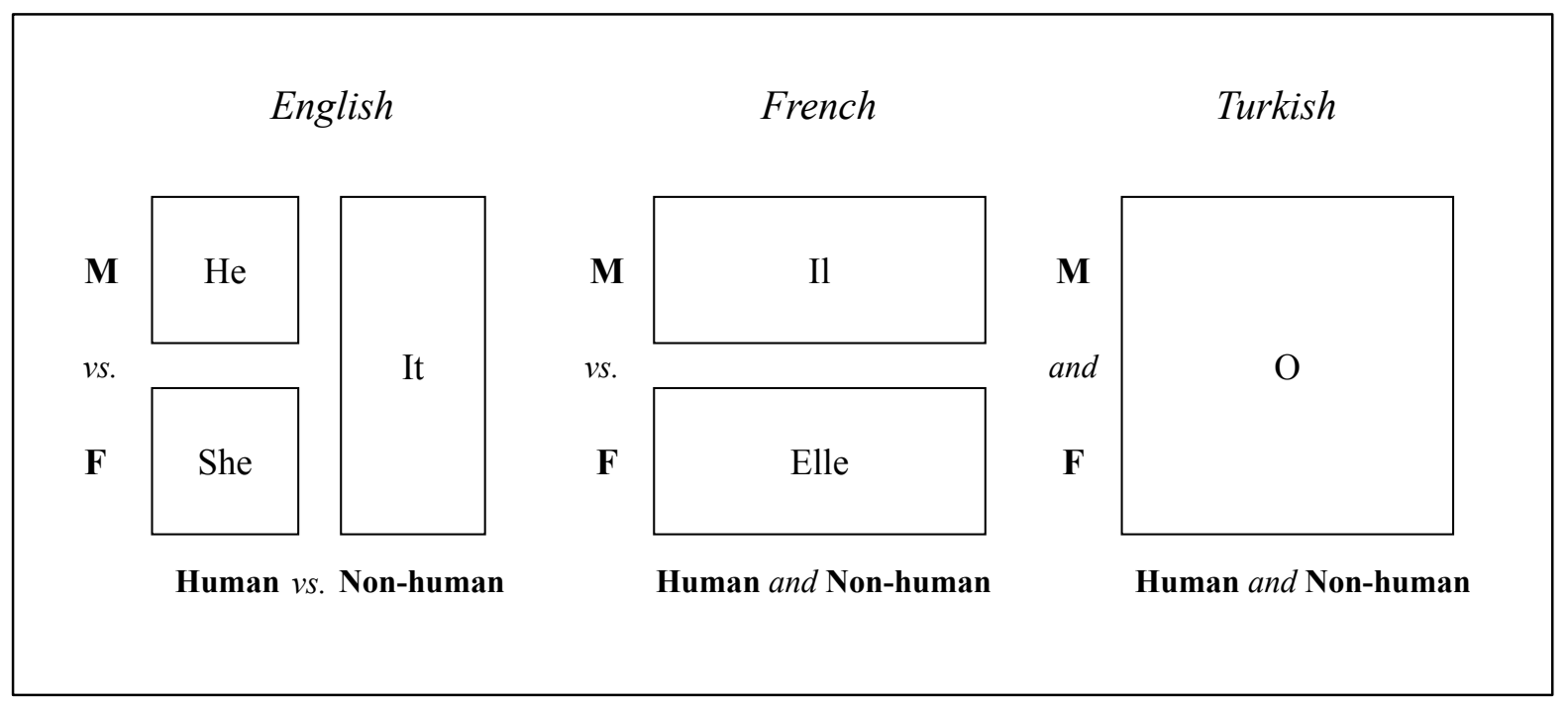

Grammatical gender affects thoughts and behaviors. In particular, how people talk about objects can influence their representations of those objects. For example, assignment of gender markers to non-human entities is typically semantically arbitrary, ${ }^{1}$ and thus differs across gendered languages: the word for key is feminine in Spanish but masculine in German, whereas the word for bridge is masculine in Spanish but feminine in German. Although these differences are arbitrary, grammatical gender affects how people represent the non-human entities. For example, when native Spanish and German speakers were asked to spontaneously come up with three adjectives to describe a key, German speakers came up with adjectives carrying masculine connotations (e.g. heavy, hard, metal), whereas Spanish speakers generated feminine adjectives to describe a key (e.g. shiny, lovely, tiny; Boroditsky, Schmidt, and Phillips 2003). Thus, it appears that despite the arbitrariness of the gender markers, they influence the gender-related traits that are ascribed to the non-human entities by leading individuals to selectively attend to an object's masculine or feminine qualities.

\footnotetext{
${ }^{1}$ Indeed, similar words for the same general concept can have different gender markings. In French, coronavirus is masculine (le coronavirus) but COVID-19 is feminine (la COVID-19).
} 
Grammatical gender also affects relations between men and women. For example, compared to genderless languages, cultures with gendered languages exhibit greater gender prejudice (DeFranza et al. 2020) and less gender equality (Prewitt-Freilino, Caswell, and Laakso 2012). Like the English versus Korean example of different language treatment of motion noted earlier, the presence (vs. absence) of gender markers affects encoding and retrieval, resulting in different accessible knowledge structures. The presence of gendered markers directs attention to gender distinctions and makes them more salient (Boroditsky et al. 2003), and these processes can occur nonconsciously (Boutonnet, Athanasopoulos, and Thierry 2012).

\section{Grammatical Gender and Anthropomorphism}

We propose that a language's grammatical gender can also affect the degree to which people anthropomorphize non-human entities. However, we propose a slightly different process than the one underlying the effects of grammatical gender on gender prejudice and inequality. In our theorizing, the key distinction is not simply the presence of gendered markers per se, but also whether the language's grammar system makes a distinction between humans and non-humans. As figure 1 shows, both English and French assign gender markers to some objects, but only French assigns them to all objects, and thus does not distinguish between humans and nonhumans. In contrast, Turkish does not assign gender at all, but consequently, like French, does not grammatically distinguish between humans and non-humans. Given that the key grammatically distinguishing feature of French and Turkish is that they do not have a separate pronoun $i t$ to grammatically mark nouns, for simplicity, we refer to such languages as it-less 
languages, and correspondingly to differentiate, we refer to languages such as English as non-itless languages. ${ }^{2}$

We propose that the extent to which the grammatical gender system of a language distinguishes between humans and non-humans affects the extent to which speakers of those languages anthropomorphize. Given that the grammatical gender system of a language can affect the accessibility of knowledge structures, we expect that speakers of languages that do not grammatically distinguish between humans and non-humans (e.g., French) will be more likely to rely on human knowledge structures when reasoning about non-human entities compared to those whose language does make this distinction. In languages with this distinction, humans and non-humans not only belong to different categories, but there is also a hierarchy between these categories, which puts humans above non-humans. Thus, we hypothesize that native speakers of languages that lack a specific pronoun for non-humans (it-less languages) are more likely to attribute human qualities to non-humans than those whose native language makes the grammatical distinction between human and non-human pronouns (non-it-less languages).

Because culture and language are intertwined, one fundamental difficulty is demonstrating that language is indeed the driver of differences in anthropomorphism. To clearly demonstrate the effect, we also investigate effects within it-less languages. If speakers of it-less languages are more likely to anthropomorphize than speakers of non-it-less languages, the way speakers of it-less languages anthropomorphize and interact with an object should depend on the grammatical properties of the object, such as the grammatical gender. Given that consumers with different beliefs and expectations perceive the same anthropomorphized object in different ways (Kim and McGill 2011), we argue that the grammatical gender of non-human objects in it-less

\footnotetext{
${ }^{2}$ Although the terms are less than elegant, no formal linguistic term exists to make this distinction. Thus, we chose this "linguistic shorthand" to increase the fluency of the prose.
} 
languages will affect consumers' judgements, even though the grammatical gender assigned to non-human entities is semantically arbitrary. Moreover, because speakers of gendered languages know that the assignment of grammatical gender is arbitrary, they would not consciously use grammatical gender to form judgments; thus, we expect individuals to be unaware of the effect of gender marking on their judgments (Boutonnet et al. 2012).

\section{EMPIRICAL OVERVIEW}

Across 10 studies, we test the proposition that aspects of language influence the relationships that consumers have with products. More specifically, we test the proposition that consumers whose native language does not grammatically differentiate between human and nonhuman entities (it-less) anthropomorphize more than those whose language has a separate pronoun for non-humans (non-it-less). In the first six studies, we provide a comprehensive test of the general proposition that language influences anthropomorphism tendencies. Studies 1A and $1 \mathrm{~B}$ test the proposition that speakers of an it-less language-Turkish in study $1 \mathrm{~A}$ and French in study 1B-anthropomorphize more compared to speakers of a non-it-less language (English). Study 1C tests the same proposition using a more consequential measure of anthropomorphism (categorization task). Study 2 relies on archival market data from six countries to test whether the predicted pattern exists for the consequences of anthropomorphism (product disposal behavior). Studies 3A and 3B address issues of causality by manipulating pronoun usage through a learning task in which English-speaking participants are trained to avoid the pronoun it when referring to non-human entities (and thus training them to use gendered pronouns). 
The remaining four studies explore the idiosyncratic consequences of language on anthropomorphism in consumer settings. Studies $4 \mathrm{~A}$ and $4 \mathrm{~B}$ test the proposition that consumers who speak an it-less language rely on linguistic cues (grammatical gender) in the way they attribute human characteristics to brands, and shows that attitudes are more favorable and willingness to pay is greater when the grammatical gender of the product is congruent with the gender of the participant. Study 5 replicates the effect with consequential choice. Finally, study 6 shows that the gender congruency effect reverses when interaction with the product is anticipated, and the effect is attenuated when anthropomorphism is explicitly primed.

In all studies, participants provided informed consent, and we analyzed the data only after all measures had been collected. We excluded participants based on a priori rules (described within each study) that were applied before any data analyses. All measures and manipulations are provided in the web appendix, and all stimuli and raw data are posted anonymously at https://osf.io/5gqy9/?view only=03d8e826680e4327af44e7561acaa55b.

\section{STUDY 1A: TURKISH VS. ENGLISH}

Study $1 \mathrm{~A}$ tested the hypothesis that native speakers of an it-less language are more likely to attribute human characteristics to non-humans than are native speakers of a non-it-less language. To do so, we compared native Turkish and English speakers' chronic tendency to anthropomorphize non-human entities. English has a specific pronoun for non-humans (it) but Turkish does not.

Method 
Participants, Design, and Procedure. One hundred forty-five participants completed the study. Participants were either native English speakers ( $n=75 ; 50$ women, 25 men; $M_{\text {age }}=38.3$ years, $S D=14.01)$ or native Turkish speakers $\left(n=70 ; 35\right.$ women, 34 men, 1 other; $M_{\text {age }}=21.9$ years, $S D=1.77$. The English-speaking participants were recruited from the Prolific Academic UK online research panel and received $£ 0.20$ as compensation; the Turkish-speaking participants were students at a major English-speaking public university in Istanbul who received course credit as compensation. The design was a one-factor study in which native language (English vs. Turkish) was measured.

As a cover story, participants were told that they would be participating in a short study about the way they perceive themselves and others in various situations. Participants first completed the 15-item Individual Differences in Anthropomorphism Questionnaire (IDAQ; Waytz et al. 2010), measured along an 11-point scale $(0=$ strongly disagree; $10=$ strongly agree; $\alpha=.846$; see web appendix A for the complete list of items). Both English and Turkish participants answered the online questionnaire in English to make the test more conservative and prevent any language priming effects (Chen and Bond 2010). Next, participants answered the attention check question (if you are reading this, please select 3), and then went through a second unrelated study that was part of a separate research project. Finally, participants reported their age, gender, fluency in English (on an 11-point scale: $0=$ not at all fluent, $10=$ fluent), and native language. Fluency and native language were used as exclusion criteria. In this study, and all that follow, neither age nor gender influenced the hypothesized effects, and thus are not discussed further. 
Results and Discussion

Exclusion Criteria. All participants passed the attention check, and also were either native English speakers or scored sufficiently high on the language fluency measure to pass this criterion. Thus, no data were excluded from the analyses.

Hypothesis Testing. We expected that Turkish participants would exhibit higher levels of chronic anthropomorphism (and thus score higher on the IDAQ) than would English participants. A one-way ANOVA confirmed our expectations. Native Turkish speakers $(M=4.04, S D=1.51)$ scored higher on the IDAQ than did native English speakers $(M=3.38, S D=1.55 ; F(1,143)=$ 6.92, $\left.p=.009, \eta^{2}=.046\right)$.

The results of study 1A provide initial support for our proposition that speakers of languages lacking a specific pronoun for non-humans are more likely to attribute human characteristics to non-humans than those whose native language grammatically differentiates between human and non-human pronouns. In addition, given that the Turkish language does not assign gender to nouns, but also does not grammatically distinguish between humans and nonhuman entities (it-less), the results suggest that the effects are driven by the it-less distinction rather than grammatical gender marking. In study 1B, we address some obvious alternative explanations for this observed relation.

\section{STUDY 1B: FRENCH VS. ENGLISH}

Although the comparison between Turkish and English speakers observed in study 1A provides initial evidence for the effects of language on the tendency to anthropomorphize, there 
are plausible alternative explanations for the results. One is that factors other than language may explain why one culture exhibits greater anthropomorphism than does another (e.g., cultural elements and scale usage). The second possible alternative explanation is that the language of administration may play a role. In study $1 \mathrm{~A}$, the questionnaire was administered in English to native Turkish speakers who were fluent in English. On the one hand, this may be viewed as a conservative test, because answering (and thinking) in a particular language may activate the knowledge structures of that language (Luna and Peracchio 2008). Thus, completing the study in English, which grammatically distinguishes between humans and non-humans, may reduce anthropomorphism for Turkish speakers. On the other hand, answering questions in a second language may affect responses (e.g., through increased anxiety) and can result in higher scores (MacIntyre and Gardner 1994). To address these concerns, we used a different language comparison (French vs. English), a larger pool of participants, and manipulated whether the language of administration was English or French for native French speakers. French and English were contrasted because English has a specific pronoun for non-humans, whereas French does not. Finally, religion is associated with anthropomorphism (Epley et al. 2007; Guthrie 2014), and thus differences in religiosity may have influenced the results. In this study, we use two cultures (French and American) that are relatively more similar in terms of religion than Turkish and British.

Method

Participants, Design, and Procedure. Two hundred twenty-five participants completed the study. Participants were either native English speakers ( $n=79 ; 39$ women, 39 men, 1 other; 
$M_{\text {age }}=33.9$ years, $\left.S D=12.88\right)$ or native French speakers $(n=146 ; 93$ women, 48 men, 5 other; $M_{\text {age }}=22.6$ year, $S D=3.20$ ). The English-speaking participants were recruited from the US Amazon Mechanical Turk (MTurk) online panel and received US\$0.50 as compensation; the French participants were recruited from a research panel of a research lab in France in exchange for a chance of winning $€ 30$ in a lottery. The design was a one-factor study in which native language (English vs. French) was measured.

We administered the IDAQ scale to either native English-speaking participants $(\alpha=.852)$ or native French-speaking participants, and also manipulated (with random assignment) whether native French-speaking participants answered in French $(\alpha=.852)$ or English $(\alpha=.828)$. For the French version of the IDAQ scale, two independent translators fluent in both languages translated the scale into French. Further, within the IDAQ scale items, we imbedded an attention check (select the number that is the sum of 2 and 3). Participants then indicated their age, gender, native language, and fluency in English.

Results and Discussion

Exclusion Criteria. Seventeen participants failed the attention check measure and thus were excluded from analyses. The data were analyzed for the remaining 208 participants.

Hypothesis Testing. First, to determine whether language of administration affected the results, we compared the scores of French speakers who completed the study in English with French speakers who completed the study in French. Language of administration did not significantly affect the results. The IDAQ scores of French participants who answered the survey in English $(M=3.76, S D=1.52)$ did not differ from the IDAQ scores of French participants who 
answered the survey in French $\left(M=3.57, S D=1.48 ; F(1,144)=.59, p=.442, \eta^{2}=.004\right)$. Thus, we collapsed the IDAQ scores for French participants across language administration conditions. Consistent with predictions, native French speakers scored higher on the IDAQ scale of chronic anthropomorphism $(M=3.67, S D=1.50)$ than did English speakers $(M=2.89, S D=$ $\left.1.61 ; F(1,206)=11.01, p=.001, \eta^{2}=.051\right)$. These findings replicate those of study $1 \mathrm{~A}$. Using a different it-less language, the results again show that individuals whose language does not have a specific pronoun for non-humans (French) anthropomorphize more than individuals whose language (English) does have a specific pronoun for non-humans. In addition, the two cultures that were compared (French and American) are relatively more similar in terms of religion compared to study 1A (Turkish and British), which alleviates concerns regarding religion as an alternative explanation.

\section{STUDY 1C: CATEGORIZATION OF HUMANS AND NON-HUMANS}

In the first two studies, we used the same measure of anthropomorphism (IDAQ scores) to test our hypotheses. In this study, we use a different measure of anthropomorphism, and a different comparison population, to establish external validity, and demonstrate that the findings are not specific to the IDAQ scale. Specifically, we operationalized anthropomorphism as the extent to which people are prone to put humans and non-humans in the same category. Categorization influences the way consumers search and retrieve information, draw inferences, and make choices (Alba and Hutchinson 1987; Cohen and Basu 1987; Sujan and Deklava 1987), and thus the task can be regarded as a theoretically consequential measure of anthropomorphism. 
In this study, we specifically test whether the way British and French participants categorize human and non-human entities reflects the differences in their pronoun systems. In terms of language comparison, we compare French native speakers with British native English speakers, but with an additional criterion that the native English speakers are bilingual and fluent in French, and the study was administered in French. Although, all British participants were bilingual in English and French, they were coordinate bilinguals, meaning that they learned French later in life, and therefore their bilingualism is not balanced. That is, although they can understand and speak French, they are more proficient in English and more likely to stick to their native language when making categorization judgements (Li, Zhang, and Nisbett 2004).

Method

Participants, Design, and Procedure. Four hundred nineteen participants completed the study. Participants were either British and native English speakers (106 women, 108 men, 4 other; $M_{\text {age }}=35.82, S D=10.76$ ), or French and native French speakers (98 women, 102 men, 1 other; $M_{\text {age }}=22.82, S D=3.82$ ). All British participants were bilingual in English and French. British participants were members of the Prolific online panel and were paid £0.20; French participants were recruited from a participant panel of a major research lab in Paris and paid $€ 4$. Both groups completed the task in French. The design was a one-factor study in which native language (English vs. French) was measured.

Participants completed the categorization task (described next), which served as our measure of anthropomorphism. Following the categorization task, participants were asked to answer a series of questions that included an attention check question, which also served as a 
measure of French proficiency. The question was posed in French "Combien de lettres y a-t-il dans le mot lettre?" (How many letters are there in the word letter?). Participants then indicated their age, gender, mood, and native language. Mood did not affect the results, and thus is not discussed further.

Categorization Task. People organize and classify objects based on perceived attribute similarities, which is the basis of taxonomic categorization. For example, if people are given the quadruplet man/table/woman/piano, and are asked which two go together, the choice man/woman and table/piano suggests a taxonomic categorization organized around a humannon-human distinction (animacy dimension). However, cognition and reasoning styles differ across cultures, and language can influence the way people group objects ( $\mathrm{Ji}$ et al. 2004). Another viable grouping for French speakers is to categorize by grammatical gender. Thus, consistent with our theoretical reasoning, French speakers may be more likely to choose a categorization such as man/piano and woman/table, because the French language assigns the masculine grammatical gender to man and piano, and the feminine grammatical gender to woman and table. Accordingly, we expect that native French speakers will be more likely than will native English speakers to categorize by grammatical gender, whereas native English speakers will be more likely to categorize by animacy than will native French speakers.

To test this proposition, participants were given a list of 12 nouns and asked to form two categories according to their criterion of choice. The presentation order of the nouns was randomized. Of those nouns, we crossed whether they were animate or inanimate, and whether their grammatical gender (based on the article) was masculine or feminine. Thus, six were animate and six were inanimate; six had a masculine grammatical gender and six had a feminine grammatical gender (see web appendix C). Participants dragged the randomly listed 12 nouns 
into two pre-designed boxes that were labeled as Category One and Category Two. Based on the way participants categorized the 12 nouns, we labeled their categorization as based on the gender of the nouns, based on the animacy dimension, or neither (see web appendix $\mathrm{C}$ for full details of scoring).

Results and Discussion

Exclusion Criteria. Data from 18 British participants who failed the test evaluating their French proficiency were excluded from analyses. The data were analyzed for the remaining 401 participants.

Hypothesis Testing. We expected that native French speakers would be more likely to categorize based on the grammatical gender of the (non-human) noun compared to native English speakers. The two groups differed significantly in the way they did the categorization $\operatorname{task}\left(\chi^{2}(2)=20.57, p<.001\right.$; see figure 2). Consistent with predictions, French speakers categorized more on grammatical gender (32.8\%) than did English speakers $(14 \% ; z=4.452, p<$ $.001)$, whereas English speakers categorized more on animacy (62.5\%) than did French speakers $(51.7 \% ; z=2.177, p=.029)$. Although unrelated to our hypotheses, English speakers were more likely to fall into the Neither category (23.5\%) compared to French speakers $(15.5 \% ; z=2.043, p$ $=.041)$. However, excluding the participants in the Neither category did not change the results. French speakers were still more likely to categorize by grammatical gender (38.8\%) compared to English speakers $\left(18.3 \% ; \chi^{2}(1)=16.44, p<.001\right)$.

FIGURE 2 
GRAMMATICAL GENDER VS. ANIMACY CATEGORIZATION AS A FUNCTION OF

\section{LANGUAGE (STUDY 1C)}

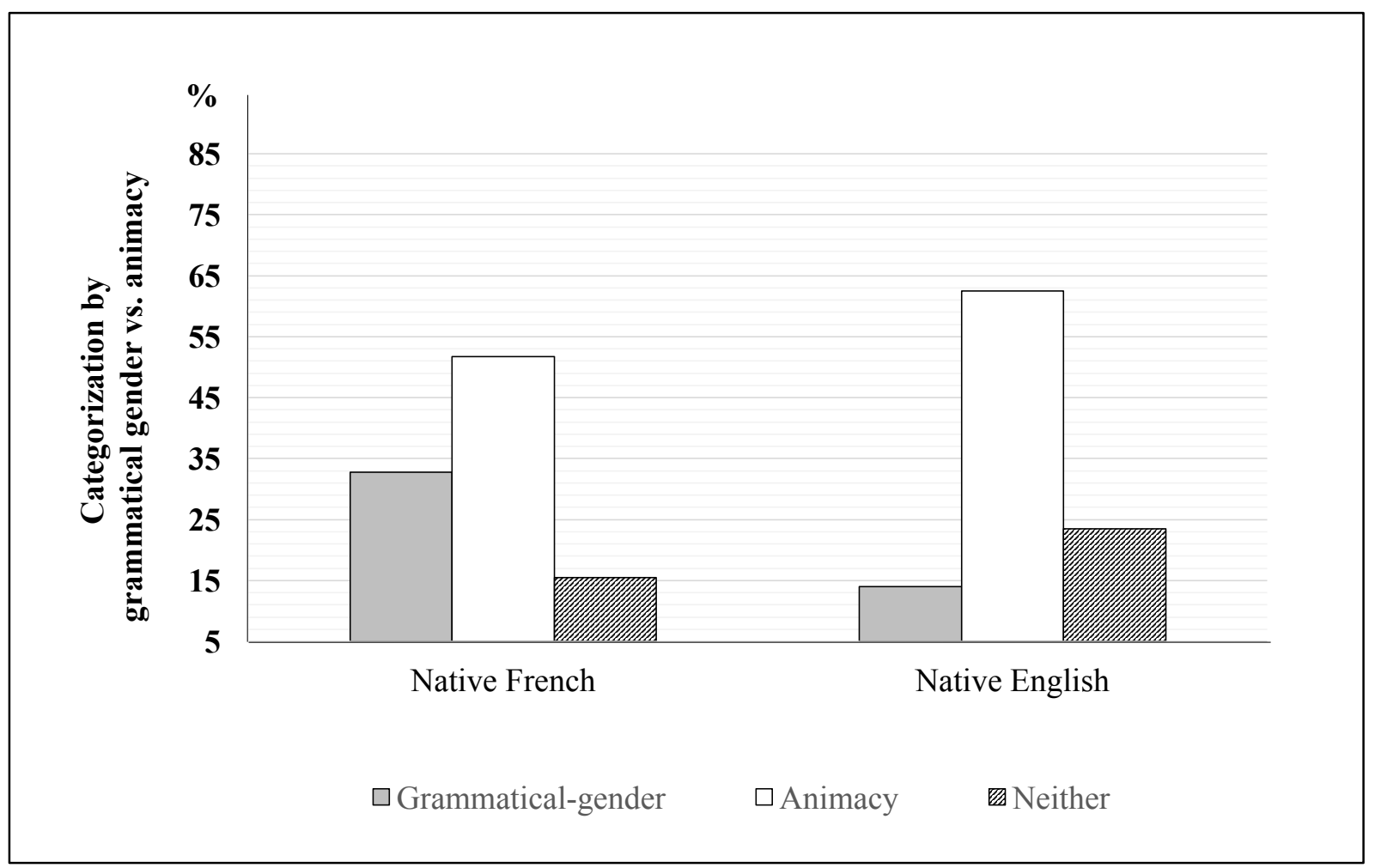

The results of study $1 \mathrm{C}$ provide further evidence of language effects on anthropomorphism. The results of the previous two studies demonstrated that language is associated with anthropomorphism based on scores on the IDAQ scale. In this study, we showed that language also affects the categorization process. Speakers of an it-less language (French) were more likely to categorize based on grammatical gender, and less likely to categorize based on animacy, than were speakers of a non-it-less language (English). Thus, the way English and French participants categorized human and non-human entities reflected the differences in their pronoun systems. The French pronoun system, unifying the conceptual universe for humans and non-humans, made native French speakers more likely to put humans and non-humans into the same category, compared to native English speakers. As an it-less language, yet a gendered one, 
native French speakers focused more on the grammatical gender when categorizing humans and non-humans than did native English speakers.

\section{STUDY 2: LANGUAGE AND SECOND-HAND SALES}

The first three studies demonstrated that speakers of it-less languages have greater anthropomorphic tendencies compared to speakers of non-it-less languages. In this study, we investigate how language-induced anthropomorphism tendencies may influence product disposal behavior. When consumers anthropomorphize products, they form connections with the products that resemble interpersonal relations, which reduces the likelihood that they will replace the anthropomorphized product (Chandler and Schwarz 2010). Thus, if language influences anthropomorphic tendencies in the way that we have theorized, then consumers who are native speakers of it-less languages should be less likely to sell their products compared to consumers who are speakers of non-it-less languages. If so, then the market for second-hand goods should be smaller in countries whose language is it-less compared to the market for second-hand goods in countries whose language is non-it-less, resulting in less likelihood of buying second-hand goods in it-less versus non-it-less language countries. To test this proposition, we used archival country-level data for the second-hand product market from six countries $(N=14,478)$, three whose language is it-less and three whose language is non-it-less.

Method 
Dataset. We used the data from the 2019 Statista Global Consumer Survey to generate our dataset. The Statista survey is conducted annually to provide a global perspective on consumption, covering both online and offline channels. The survey covers consumers' consumption activities for a wide variety of industries. Of interest to our research, one question asks "Which of these articles have you bought second hand in the past 12 months (no matter if online or in person)?" The options featured 12 product categories and the option to indicate no second-hand purchase ("I did not buy anything second hand").

The survey results concerning consumers' second-hand market purchase activities were available for eight countries: the US $(n=4210)$, the UK $(n=2038)$, Canada $(n=2056)$, France $(n=2050)$, Italy $(n=2069)$, Spain $(n=2055)$, Germany $(n=2078)$, and Austria $(n=2070)$. We selected the first six countries for our analysis. (The selection criteria were made prior to any analyses). For the US, UK, and Canada, the native language is English (non-it-less). For the next three (France, Italy, and Spain), the languages have gendered pronouns for non-humans, and thus they do not grammatically distinguish between humans and non-humans (it-less languages). We excluded Austria and Germany from our analysis because their native language is German, which has both gendered (der, die) and non-gendered (das) nouns. Thus, we pooled the data for France, Italy, and Spain into one group (it-less languages), and the US, UK, and Canada into one group (non-it-less languages). We then compared the proportion of consumers who indicated they did not buy anything second-hand between the two groups.

Results and Discussion 
Hypothesis Testing. We expected that consumers in France, Italy, and Spain $(n=6174)$ should be less likely to buy second-hand products compared to consumers in the US, UK, and Canada $(n=8304)$. The difference in proportions was significant $(z=-18.31, p<.001)$. Consistent with predictions, in France, Italy, and Spain, the proportion of consumers who bought second-hand products (35\%) was significantly lower than the proportion of consumers who bought second-hand products in English-speaking countries (50.3\%).

This study extends the findings of Chandler and Schwarz (2010), and replicates our findings from the first three studies with a more consequential measure of anthropomorphism. Although there are likely a number of alternative explanations for the country differences in buying second-hand products, the findings are nevertheless consistent with our theorizing.

\section{STUDY 3A: THE IMPACT OF LEARNING AN IT-LESS LANGUAGE ON ANTHROPOMORPHISM TENDENCIES}

The previous studies compared levels of anthropomorphism as a function of culture and associated language. Because the designs of the studies were necessarily correlational, they raise issues of causality: there may be a number of reasons why cultures differ on anthropomorphism, independent of the effect of language. To address this issue, study $3 \mathrm{~A}$ manipulated the usage of pronoun categories of native English speakers through a learning task (Roberson et al. 2005) to test the proposition that pronoun categories affect people's tendency to anthropomorphize. We expect that training native English speakers to use pronouns like native French speakers (using gendered pronouns for non-human entities) will increase their propensity to anthropomorphize. 


\section{Method}

Participants, Design, and Procedure. One hundred twenty members of the MTurk (US) online panel ( 57 women, 63 men; $M_{\text {age }}=36.5$ years, $\left.S D=9.95\right)$ participated in the study in exchange for US\$1.00. Participants were randomly assigned to conditions in a one-factor (learning task using it as pronoun: yes, no) between-subjects design. Participants first completed the learning task that served as the manipulation of pronoun usage. All participants rewrote 60 sentences by replacing an underlined noun with a pronoun. In the control group (using the pronoun it in the usual way for English), participants replaced the nouns with regular pronouns (he/she/it). For example, participants transformed the sentences in the following way: The apple pie was delicious. $\rightarrow$ It was delicious. / Jane ate the apple. $\rightarrow$ She ate the apple. In contrast, in the treatment group, participants were instructed to not use the pronoun it. Instead, they were instructed to use either he or she, depending on the arbitrary grammatical gender indicated in parentheses next to the underlined noun. For example, they transformed sentences in the following way: The apple pie was delicious. $\rightarrow$ She was delicious. / Jane ate the apple. $\rightarrow$ She ate the apple. Following the learning task, participants completed the IDAQ scale. The way participants used the pronouns in the treatment group mimics the French language, in which speakers use $i l$ (he) or elle (she) to refer to non-humans (see web appendix D for full details).

Results and Discussion

Exclusion Criteria. Data from 13 participants who did not follow the instructions of the learning task were excluded from analyses. The excluded participants either did not correctly rewrite the sentences replacing the pronouns or did not rewrite the sentences at all, and 
exclusions were equally distributed between the two conditions. Thus, the data were analyzed for the remaining 107 participants.

Hypothesis Testing. We expected participants in the treatment condition, who did not use the pronoun $i t$, would score higher on the IDAQ scale than those in the control condition. Consistent with our prediction, a one-way ANOVA indicated that participants in the treatment group $(M=3.12, S D=1.38)$, who used pronouns like a native French speaker (third-person singular pronouns did not include it for non-human entities), anthropomorphized more than did those in the control group $\left(M=2.57, S D=1.26 ; F(1,105)=4.63, p=.034, \eta^{2}=.042\right)$, who used all English third-person singular pronouns (he/she/it).

The results of study $3 \mathrm{~A}$ replicate those of the first four studies, again showing that speakers of an it-less language are more likely to anthropomorphize than are speakers of a nonit-less language. In addition, we addressed issues of causality by training some native Englishspeaking participants to avoid using the pronoun it for non-human entities, but instead to assign a gendered pronoun (he, she) as native French speakers do. However, one limitation of study $3 \mathrm{~A}$ is that the connection between the learning task pronoun usage task and anthropomorphism may have been too overt, and thus guided participants toward responses on the IDAQ that supported our hypotheses. To address this issue, in study 3B, we again use the same learning task but with a more subtle measure of anthropomorphism to reduce concerns about potential demand cues.

\section{STUDY 3B: THE IMPACT OF LEARNING AN IT-LESS LANGUAGE ON OBJECT DESCRIPTIONS}


In study $3 \mathrm{~B}$, we replicate study $3 \mathrm{~A}$ with a different measure of anthropomorphism to demonstrate that the findings are not specific to the IDAQ scale. Specifically, we used the Heider-Simmel illusion, which is an animated film of simple moving shapes (Heider and Simmel 1944), and asked participants to describe what they watched. Using text analysis on individuals' descriptions of moving shapes, we tested whether the same learning task that we used in study 3A increases individuals' tendency to ascribe human characteristics to the moving shapes. This measure has been successfully used in prior research on anthropomorphism (Fussell et al. 2008; Scheelea et al. 2015).

Method

Participants, Design, and Procedure. One hundred and forty-seven native Englishspeaking members of the Prolific Academic UK online research panel (109 women, 37 men, 1 other; $M_{\text {age }}=35.8$ years, $S D=12.74$ ) participated in the study in exchange for $£ 1.50$. The design was the same one-factor design used in study $3 \mathrm{~A}$, and participants were randomly assigned to the pronoun usage conditions (learning task using it as pronoun: yes, no). Participants were told that they would be completing two different studies. In the first study, ostensibly about understanding the pronoun use of native English, participants first completed the learning task, which was similar to the one used in study $3 \mathrm{~A}$ (see web appendix E for full details). Next, in a second study ostensibly about visual information recollection, they watched the one-minute version of the Heider-Simmel illusion of simple geometric shapes, and then wrote a description of what they saw in the video. Finally, they provided demographic information. 
Heider-Simmel Illusion Task. In this task, participants watch an abstract film of simple geometrical shapes moving around (Heider and Simmel 1944). Viewers commonly attribute human characteristics, such as emotional states, to these shapes when they are asked to describe their movements (Heberlein and Adolphs 2004). In line with previous research that has used this task to measure anthropomorphism tendencies (Scheelea et al. 2015), we used the extent to which participants attributed emotional states to the moving shapes as a proxy of anthropomorphism (Fussell et al. 2008). We coded each description by using the computer program Linguistic Inquiry and Word Count 2015 (LIWC2015) with the internal default English dictionary file and we used a previously established dictionary (Tausczik and Pennebaker 2010) to quantify the level of emotional attribution in each narrative.

Results and Discussion

Exclusion Criteria. Data from 24 participants who did not follow the instructions of the learning task were excluded from analyses. The excluded participants either did not correctly rewrite the sentences replacing the pronouns or did not rewrite the sentences at all. Additionally, we excluded four participants because they had a technical problem and could not watch the 1minute video of moving shapes. The data were analyzed for the remaining 119 participants.

Hypothesis Testing. We expected that participants in the treatment condition, who did not use the pronoun it, would attribute emotional states to the shapes more so than would those in the control condition. Consistent with our prediction, a one-way ANOVA indicated that participants in the treatment group $(M=2.98, S E=.38)$ anthropomorphized more than did those in the control group $\left(M=1.88, S E=.38 ; F(1,117)=4.14, p=.044, \eta^{2}=.034\right)$. These results replicate 
the findings of study $3 \mathrm{~A}$ with a different and more subtle measure of anthropomorphism, and also show that the findings are not specific to the IDAQ scale.

In the first six studies, we provided support for the hypothesis that speakers of languages whose grammatical gender systems do not distinguish between humans and non-humans (it-less languages) will anthropomorphize more than speakers of languages that do make this distinction (non-it-less languages). We did so by comparing anthropomorphism tendencies as a function of natural differences in languages (e.g., French, English, Turkish) and by manipulating pronoun use via a learning task. In the next four studies, we demonstrate the consequences of this effect on consumer judgments.

\section{STUDY 4A: GRAMMATICAL GENDER AND ANTHROPOMORPHISM}

In study 4A, we test whether the way speakers of it-less languages anthropomorphize and interact with an object (product) depends on the grammatical properties of non-human entities. Given that native French speakers regard grammatical gender as a classifier, which causes them to put humans and non-humans into the same category (study 2), and that they are more prone to anthropomorphism (study 1B), we test the extent to which the grammatical gender of a consumption object (e.g., brand, product) affects consumers' relations with and evaluations of those objects.

People apply social categorization processes to anthropomorphized entities, and in doing so they show an in-group bias towards human-like entities that have similar characteristics as themselves (Eyssel and Kuchenbrandt 2012). Thus, when asked to evaluate products, we expect that native French consumers will consider the grammatical gender in the way they 
anthropomorphize, but native English speakers will not. Thus, for French speakers, we expect men to have more favorable evaluations of a brand whose name takes the masculine grammatical gender than when it takes the feminine grammatical gender, and the opposite should be true for women. Put differently, for French speakers, participants will evaluate the brand more favorably when the grammatical gender of the brand name is congruent with their own gender than when it is incongruent. However, for English speakers, we expect no effects of participant gender on product evaluations.

We test this proposition in French because it is an it-less language and assigns grammatical gender to non-humans. As noted earlier, in French, each noun has a specific article that indicates the grammatical gender of the noun: le (masculine article) and la (feminine article). However, it is important to note that in the French language, for non-human entities, the gender of the article $(l e, l a)$ modifying the noun (product) is unrelated to the typical user of the product (e.g., la cravate, in which cravate means necktie, takes the feminine article; le mascara, in which mascara is an eye cosmetic, takes the masculine article).

Although every noun has a specific article, a small number of nouns can take both articles. To test our hypothesis, we identified a non-human noun (cache) that can take either the feminine (la) or the masculine (le) grammatical gender. We then used Cache as the brand name and manipulated whether it took the masculine (Le Cache) or feminine (La Cache) article.

Method

Participants, Design, and Procedure. Participants $(N=488)$ were either native English speakers $\left(n=246 ; 145\right.$ women, 101 men; $\left.M_{\text {age }}=34.5, S D=12.09\right)$ who were members of the 
Prolific UK online panel, or native French speakers $\left(n=242 ; 122\right.$ women, 120 men; $M_{\text {age }}=26.8$ years, $S D=8.31$ ) who were members of a participant pool of a research lab in Paris. British participants were paid $£ 0.20$. French participants were given a chance of winning $€ 30$ in a lottery. The experiment was a 2 (native language: English, French) $\times 2$ (participant gender: male, female $) \times 2$ (grammatical gender: masculine, feminine) between-subjects design in which grammatical gender was manipulated (random assignment) and the other two independent variables were measured.

The participants were told that they would be evaluating a billboard ad of a new clothing brand opening soon in Paris (see web appendix F). The brand name mentioned in the billboard ad was manipulated to have either a masculine (Le Cache) or feminine (La Cache) article. After seeing the ad, participants indicated their attitude toward the ad with two items (I find the ad attractive; I like the articles featured in the billboard ad), along an 11-point scale $(0=$ strongly disagree, $10=$ strongly agree). The two items were averaged to form a composite attitude measure $(r=.699, p<.001)$. Next, participants answered the attention check question (asking them to select the number 2 as their response), and then indicated their age, gender, fluency in English, fluency in French, and their native language.

Results and Discussion

Exclusion Criteria. Data from four participants who failed the attention check were excluded from analyses. The data were analyzed for the remaining 484 participants.

Hypothesis Testing. We expected that native French speakers would demonstrate an ingroup bias according to the grammatical gender of the brand, and thus give more favorable 
evaluations of the ad when the gender of the brand name was the same as their own gender (gender-congruent) compared to when the gender of the brand name was the opposite of their own gender (gender-incongruent). In contrast, we expected no effects of either participant gender or grammatical gender of the brand name for native English speakers.

To test this proposition, we conducted a 2 (native language) $\times 2$ (participant gender) $\times 2$ (grammatical gender) ANOVA with attitude towards the ad as the dependent variable. The results of this analysis can be seen in figure 3 . There was a main effect of language, with English-speaking participants evaluating the ad more favorably than French-speaking participants $\left(F(1,476)=4.94, p=.027, \eta^{2}=.01\right)$. More importantly, the predicted three-way interaction was significant, although marginally so $\left(F(1,476)=3.23, p=.073, \eta^{2}=.007\right)$.

To decompose the interaction, we conducted separate 2 (participant gender) $\times 2$ (grammatical gender) ANOVAs for French and English speakers. As expected, the grammatical gender $\times$ participant gender interaction was significant for French speakers $(F(1,238)=7.02, p=$ $\left..009, \eta^{2}=.029\right)$. As the left panel of figure 3 shows, as predicted, French-speaking men evaluated the ad more favorably when the brand name was grammatically gender-congruent (Le Cache; $M$ $=5.57, S E=.25)$ than when it was grammatically gender-incongruent (La Cache; $M=4.82, S E=$ $\left..24 ; F(1,238)=4.63, p=.032, \eta^{2}=.019\right)$. The same gender-congruence effect was also observed for women, who evaluated the ad for La Cache more favorably $(M=5.77, S E=.24)$ than the ad for Le Cache $(M=5.22, S E=.24)$, although the difference only approached significance $(F(1$, $238)=2.54, p=.11, \eta^{2}=.011$ ). In contrast, for English speakers (right panel of figure 3), neither the interaction $\left(F(1,238)=.021, p=.884, \eta^{2}<.011\right)$ nor the simple effects within both genders were significant, again consistent with predictions $(p \mathrm{~s}>.46)$. 
FIGURE 3

ATTITUDE TOWARD THE AD AS A FUNCTION OF GRAMMATICAL GENDER, PARTICIPANT GENDER, AND NATIVE LANGUAGE (STUDY 4A)

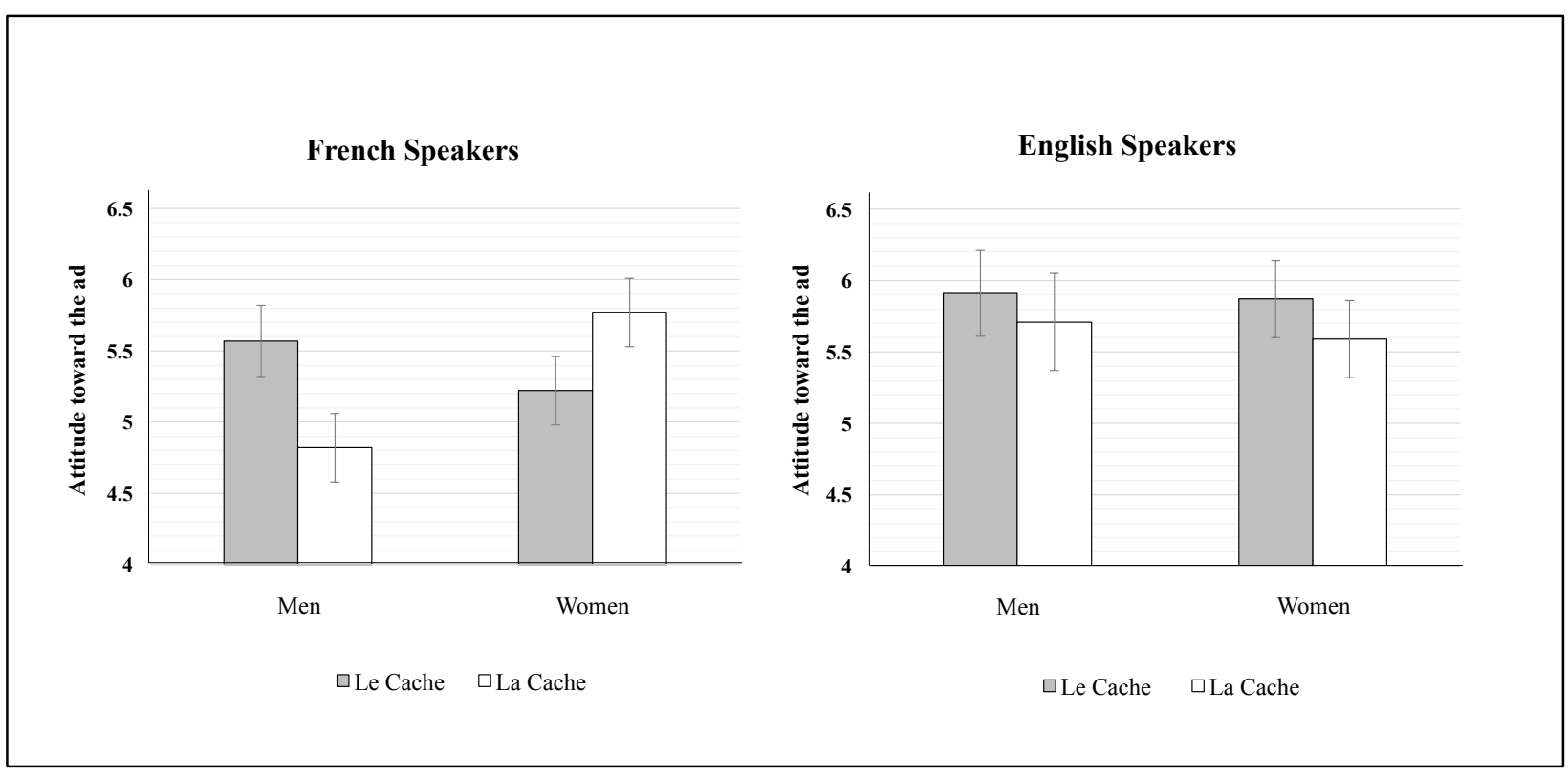

The findings of study 4A show that the grammatical gender of a brand name affects consumers' evaluations of the brand for French speakers, whose language does not grammatically distinguish between humans and non-humans. French participants evaluated an ad for the brand more favorably when the participant gender and grammatical gender were congruent compared to when they were incongruent. This finding confirms and extends research showing that people show an in-group bias towards humanlike entities that have similar characteristics as themselves (Eyssel and Kuchenbrandt 2012). In contrast, there was no effect of grammatical gender for English speakers, whose native language does distinguish between humans and non-humans.

Alternative Explanations. Although the findings of study 4A support our hypotheses, there are potential alternative explanations for the findings. One is that the results simply reflect 
a gender match in which participants' brand attitudes were based on the presumed gender of the clothing, which is reflected by the gender of the article $(l e, l a)$. That is, participants may have inferred that the brand name article indicated the type of clothing (for men or for women). However, as we noted earlier, in the French language, the gender of the article modifying the noun is unrelated to the gender of the typical user, and the ads for each brand showed both genders (web appendix F). In addition, no gender match effects were noted for native English speakers, who would arguably be more inclined to use the gender of the noun to infer the gender of the typical user of the clothing. Nevertheless, to address this issue, in study 4B, we replicate study 4A using a genderless product category (ice cream).

A second possibility is related to the meaning of the brand name used in the study. Although in French a small number of nouns can take both articles, the meaning can change slightly, depending on the article. In the case of Le Cache and La Cache, the meaning changes as a function of its gendered article (le vs. la). Le Cache means a thing that is used to hide someone or something. La Cache means a place to hide someone or something. To avoid the confound in meaning, in the next study we use a nonsense brand name rather than an existing word. Finally, a third possibility is that the articles for the brand name $(l a, l e)$ have different phonetic properties (differences in pitch), which may have influenced the findings via phonetic symbolism (Lowrey and Shrum 2007). However, the fact that we did not observe the effect with English-speaking participants further suggests that the effect is also not driven by sound symbolic differences in the brand names.

STUDY 4B: GRAMMATICAL GENDER AND ANTHROPOMORPHISMREPLICATION WITH A NONSENSE BRAND NAME 
The purpose of study 4B was to replicate the findings of study 4A with a different product (one that is gender-neutral) and a brand name that has no meaning (fictitious word). In this study, we used only French-speaking participants and the study was administered in French.

Method

Participants, Design, and Procedure. Two hundred and three native French-speaking members ( 130 women, 70 men, 3 other; $M_{\text {age }}=22.2$ years, $S D=3.18$ ) of a participant pool of a research lab in Paris completed the study in exchange for $€ 5$. The study employed a 2 (noun gender: $l a, l e) \times 2$ (participant gender: male, female) between-subjects design in which grammatical gender of the brand name was manipulated (random assignment) and participant gender was measured. Participants were told that they would be asked to complete a series of tasks. In the first task, they were told that they would be evaluating an ad for a new ice-cream shop opening soon in Paris. The brand name for the shop was a nonsense word, $\mathrm{mmm}$, and thus could take either a masculine (le) or feminine (la) article (see web appendix G). After seeing the ad, either featuring the brand name le $\mathrm{mmm}$ or la $\mathrm{mmm}$, participants indicated their willingness to purchase the ice cream, along an 11-point scale $(0=$ strongly disagree, $10=$ strongly agree $)$. Next, participants completed an unrelated study (which contained an attention check), after which they indicated their age, gender, fluency in English, and native language.

Results and Discussion 
Exclusion Criteria. No participants failed the attention check. Data from three participants who indicated a non-binary gender option (other, preferred not to answer) were excluded from analyses. The data were analyzed for the remaining 200 participants.

Hypothesis Testing. We expected that men would be more willing to purchase the ice cream when the ad featured the masculine grammatical gender $(l e \mathrm{mmm})$ than when it featured the feminine grammatical gender (la $\mathrm{mmm}$ ), but the opposite would be true for women. To test this hypothesis, we conducted a 2 (grammatical gender: $l a, l e) \times 2$ (participant gender: male, female) ANOVA with willingness to purchase as the dependent variable. The results of this analysis can be seen in figure 4. Only the interaction was significant $(F(1,196)=7.1, p=.008$, $\left.\eta^{2}=.035\right)$. As expected, men were more willing to purchase the ice cream in the masculine grammatical gender condition $(M=6.02, S E=.49)$ than in the feminine grammatical gender condition $\left(M=4.77, S E=.49 ; F(1,196)=3.3, p=.071, \eta^{2}=.017\right)$, although the difference was only marginally significant. Similarly, women were more willing to purchase the ice cream in the feminine grammatical gender condition $(M=5.51, S E=.35)$ than in the masculine grammatical gender condition $\left(M=4.48, S E=.37 ; F(1,196)=4.12, p=.044, \eta^{2}=.021\right)$.

\title{
FIGURE 4
}

\author{
PURCHASE INTENTIONS AS A FUNCTION OF GRAMMATICAL GENDER AND
}

PARTICIPANT GENDER (STUDY 4B) 


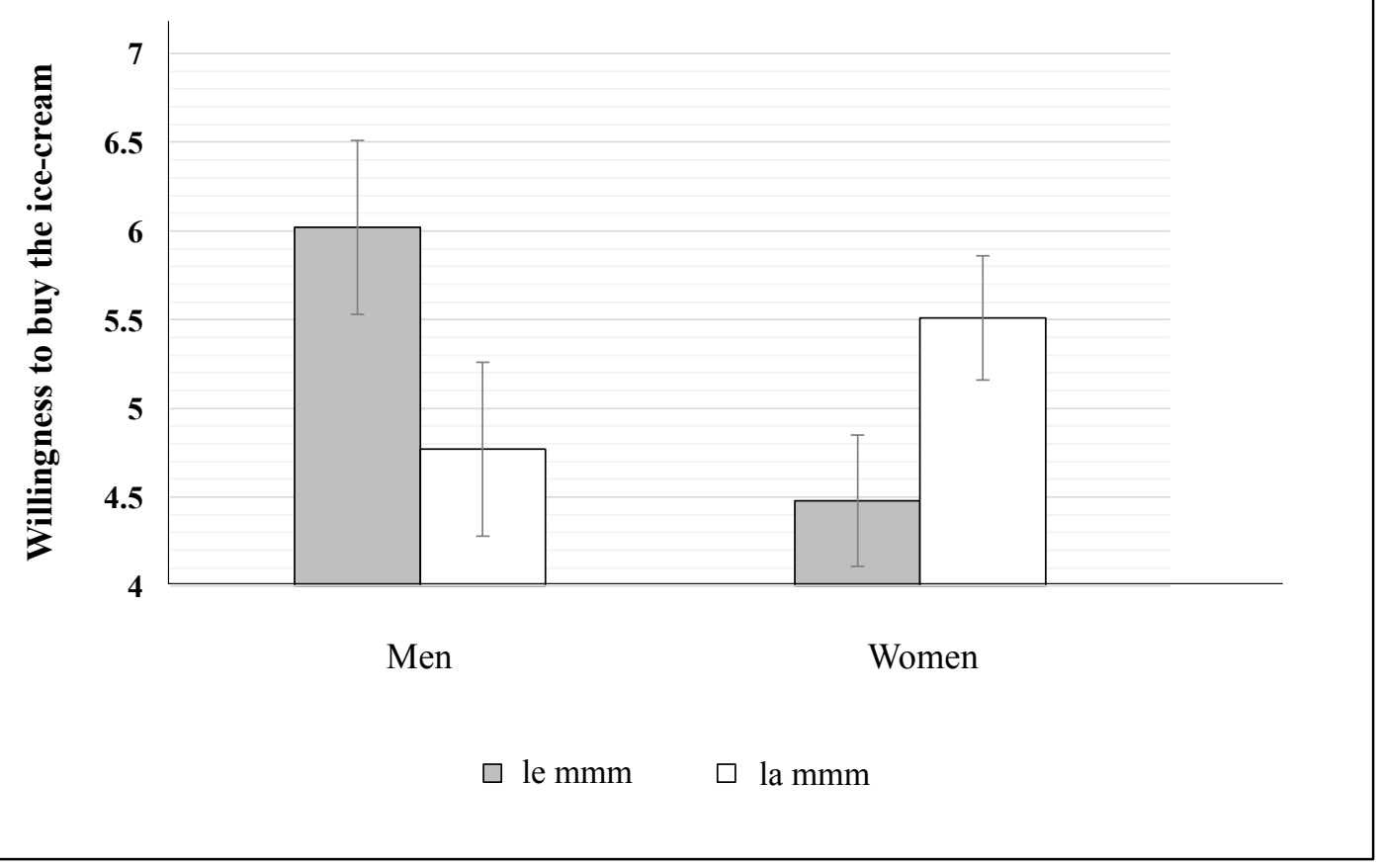

These results of study $4 \mathrm{~B}$ again show that when participants whose language contains gendered nouns are asked to form impressions about consumption objects, they consider the grammatical gender, and thus evaluate a brand more favorably when the grammatical gender is congruent with their own gender than when it is not congruent. In addition, study 4B rules out a gender match alternative explanation in which participants' brand attitudes were based on the presumed gender of the clothing, which is reflected by the gender of the article $(l e, l a)$. Using a nonsense brand name also alleviates concerns regarding the slight meaning change of the brand name in study $4 \mathrm{~A}$. 
In this study, we test the same effect in a consequential choice situation to demonstrate that even when consumers have a vested interest in the outcome, grammatical gender still influences the way they anthropomorphize and interact with non-humans (products).

Method

Participants, Design, and Procedure. Two hundred and two French-speaking members (103 women, 97 men, 2 other; $M_{\text {age }}=23$ years, $S D=3.51$ ) of a participant pool of a research lab in Paris participated in the study in exchange for $€ 4$.

The study was a 2 (noun gender: $l e, l a) \times 2$ (participant gender: male, female) mixed design in which grammatical gender was manipulated within-subjects and gender was measured. As part of the experimental setting, to mimic a real-life choice situation, we offered a small gift to all participants at the end of another experiment. Participants were told that as an additional reward for their participation, we were offering a small gift, which was in an envelope before them. There were two envelopes, and they were told to pick which one they wanted (web appendix H). The envelopes were identical except for their labels (La Cache vs. Le Cache), and order of presentation (left vs. right) was randomized. (Regardless of the envelope chosen, all participants received an extra $€ 1$.)

Results and Discussion

Exclusion Criteria. Data from two participants who indicated a non-binary gender option were excluded from analyses. The data were analyzed for the remaining 200 participants. 
Hypothesis Testing. We expected that men would be more likely to choose the envelope labeled Le Cache than the one labeled La Cache, but the opposite would be true for women. An overall Chi-square analysis indicated that choices of men and women differed $\left(\chi^{2}(1)=8.032, p=\right.$ .005). The results of this analysis can be seen in figure 5. Within gender, as expected, men chose the envelope labeled Le Cache more often than the one labeled La Cache $(60.8 \%$ vs. 39.2\%; $\left.\chi^{2}(1)=4.564, p=.033\right)$, whereas women showed the opposite pattern, choosing La Cache marginally more often than Le Cache $\left(59.2 \%\right.$ vs. $40.8 \% ;\left(\chi^{2}(1)=3.505, p=.061\right)$.

FIGURE 4

GIFT CHOICE AS A FUNCTION OF GRAMMATICAL GENDER AND PARTICIPANT GENDER (STUDY 5)

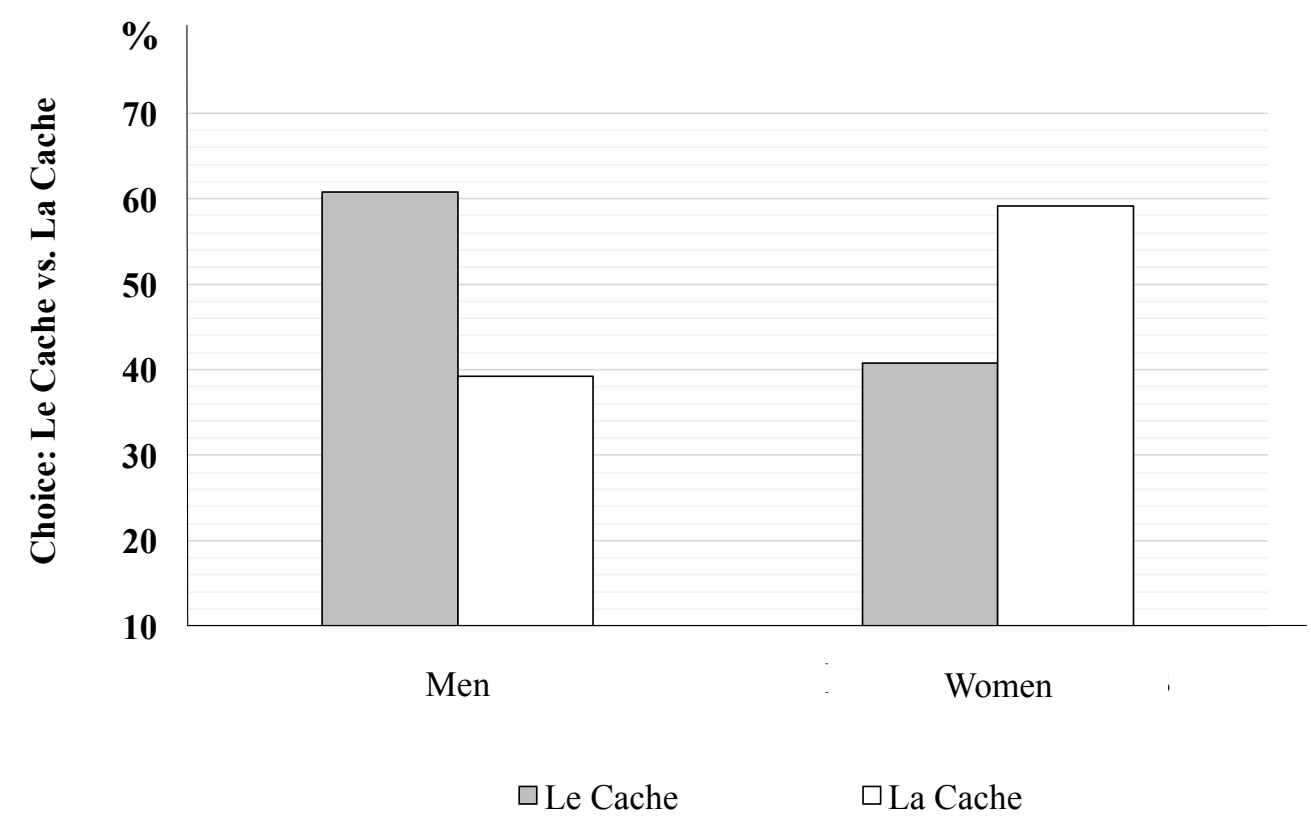


The findings of study 5 replicate those of the previous studies using actual choice as the dependent variable. In study 6, we explore boundary conditions for this effect.

\section{STUDY 6: REVERSING THE EFFECT, ELIMINATING THE EFFECT}

Study 6 had two objectives. The first was to reverse the gender-congruity effect. In the previous three studies, we showed that for it-less languages, which assign gender to all nouns and thus do not make the grammatical distinction between human and non-human entities, the grammatical gender of a consumption object (e.g., brand, product) affects consumers' relations with and evaluations of those objects. We demonstrated this by showing that even though the grammatical gender of an object is meaningless, when forming impressions of the consumption objects, men and women show an in-group bias towards human-like entities that have similar characteristics as themselves (Eyssel and Kuchenbrandt 2012). Thus, they evaluate objects more favorably when the grammatical gender is the same as their own gender.

If our theorizing is correct, then the gender-congruency effect should reverse in situations in which there is a bias towards the opposite gender. One such situation is interpersonal interactions. Research shows that when people interact with, rather than simply form impressions of, anthropomorphized robots, they prefer interactions with a robot of the opposite gender (Park, Kim, and Del Pobil 2011; Siegel, Breazeal, and Norton 2009). This finding is also consistent with research demonstrating that consumers with different expectations perceive the same anthropomorphized object in different ways (Kim and McGill 2011). Given that anthropomorphism turns relations with objects into quasi-social relations, and that situational factors can shape one's expectations in their social relations, we expect that when consumers 
interact (or anticipate interaction) with anthropomorphized agents, they will evaluate a brand more favorably when the grammatical gender of the brand is opposite of their own gender.

A second objective was to provide evidence of the nonconscious aspect of the effect. We have argued that the grammatical gender of an object serves as a natural, nonconscious prime that influences judgments in a subtle way that operates outside of consumers' conscious awareness. Thus, it-less language consumers are unaware of the influence of grammatical gender on their tendencies to anthropomorphize and on their subsequent judgments. If so, then calling attention to this bias by making anthropomorphism salient should reduce or eliminate the effect (Bargh 1992; Lombardi, Higgins, and Bargh 1987).

Method

Participants, Design, and Procedure. Participants were 485 native French-speaking members ( 258 women, 227 men; $M_{\text {age }}=22.8$ years, $\left.S D=3.69\right)$ of a participant pool of a research lab in Paris who completed the study in exchange for a chance of winning $€ 30$ in a lottery.

The study was a 2 (grammatical gender: $l e, l a) \times 2$ (participant gender: male, female $) \times 2$ (anthropomorphism prime: implicit, explicit) between-subjects design in which grammatical gender and the anthropomorphism prime were manipulated (random assignment) and gender was measured. Participants were told that they would be participating in a study to give their opinion about a new clothing store. They were asked to imagine that they received a letter from the clothing store that would soon be opening its first store in Paris. The letter asked them to "come and celebrate the opening of the store." This procedure was to ensure that there was an anticipated interaction between the consumer and the brand rather than a simple impression 
formation (see web appendix I). To enhance realism, the letter included a promotional message indicating that when consumers show the letter at the opening, they would get a $10 \%$ discount. The letter served as manipulations of both grammatical gender and the anthropomorphism prime, and the same ad used in study 4A was included at the beginning of the letter. After reading the letter, participants indicated their attitude toward the ad, completed a manipulation check, and provided demographic information.

Manipulations and Measures. The manipulation of grammatical gender was the same as in study 4a (La Cache vs. Le Cache). The explicitness of the prime was manipulated by how the letter was written. In the implicit condition the letter simply described the brand in the thirdperson ("Le Cache is a modern apparel brand..."; see web appendix I). In the explicit anthropomorphism condition, the letter was written in the first-person as if it came from the brand itself, using first-person pronouns ("I'm Le Cache, a modern apparel brand..."). Attitude toward the ad was measured with two items (I find the ad attractive; I liked the clothing articles featured in the letter $)$, measured along a 10 -point scale $(0=$ strongly disagree, $10=$ strongly agree). The items were averaged to form a composite scale $(r=.534, p<.001)$. As a manipulation check, participants indicated the extent to which they thought the brand is masculine or feminine, along a 5 -point scale $(1=$ extremely masculine, $5=$ extremely feminine $)$. Finally, the participants indicated their age, gender, mood, fluency in English, and native language.

Results and Discussion 
Exclusion Criteria. All participants passed the attention check, and thus no data were excluded from the analyses.

Manipulation Check. Recall that in the French language, for non-human entities (nouns), whether the articles modifying the nouns are masculine or feminine is unrelated to the masculinity or femininity of the entities. Thus, in the implicit priming condition, we expected no differences in judgments of the masculinity or femininity of the brand. This pattern of results would suggest that gender is not explicitly (consciously) considered when evaluating gendermarked brand names. However, we expected that the explicit anthropomorphism prime would call attention to the prime, and thus influence participants' perceptions of whether the brand was masculine or feminine.

To test these hypotheses, we conducted a 2 (grammatical gender) x 2 (anthropomorphism prime) ANOVA with masculinity/femininity ratings as the dependent variable. There was a significant main effect of grammatical gender $\left(F(1,481)=8.033, p=.005, \eta^{2}=.016\right)$, and a marginally significant interaction $\left(F(1,481)=3.111, p=.078, \eta^{2}=.006\right)$. Pairwise comparisons confirmed our expectations that in the explicit, overt priming condition, participants judged the brand to be more masculine in the masculine grammatical gender condition $(M=2.97, S E=.07)$ than in the feminine grammatical gender condition $\left(M=3.25 ; S E=.06 ; p=.002, \eta^{2}=.042\right)$. In contrast, in the implicit priming condition, judgments of masculinity/femininity did not differ between the masculine $(M=3.16, S E=.06)$ and feminine grammatical gender conditions $(M=$ 3.23; $S E=.06 ; p=.439, \eta^{2}=.002$ ). These findings suggest that native French speakers do not consciously perceive gender associations as a function of the gendered articles $(l a, l e)$.

Hypothesis Testing. Because individuals tend to prefer interactions with anthropomorphized objects whose gender is opposite of theirs, we expected that in implicit 
priming conditions, men would have more positive attitudes toward the ad when the grammatical gender was feminine than when it was masculine, and the opposite would be true for women. This pattern is opposite of the findings observed in studies 4A, 4B, and 5, in which no interaction with the brand occurred. In contrast, in explicit priming conditions, we expected this effect to be attenuated.

To test these hypotheses, we conducted a 2 (grammatical gender) $\times 2$ (participant gender) $\times 2$ (anthropomorphism prime) ANOVA with attitude towards the ad as the dependent variable. Only the three-way interaction was significant $\left(F(1,477)=4.38, p=.037, \eta^{2}=.009\right)$. The results of this analysis can be seen in figure 6 . As the left panel of the figure shows, in implicit priming conditions, men evaluated the ad more favorably when the grammatical gender was feminine ( $l a$; $M=6.17, S E=.25)$ than when it was masculine $\left(l e ; M=5.5, S E=.27 p=.069, \eta^{2}=.013\right)$, whereas women evaluated the brand more favorably when the grammatical gender was masculine $(l e ; M=6.24, S E=.24)$ than when it was feminine $(l a ; M=5.61, S E=.25 ; p=.072$, $\left.\eta^{2}=.013\right)$. Although the specific comparisons are only marginally significant, the full pattern (interaction) is consistent with predictions. In contrast, for explicit priming conditions (right panel of figure 6), there were no effects of grammatical gender on attitudes toward the brand (all $p \mathrm{~s}>.25)$.

\section{FIGURE 6}

ATTITUDE TOWARD THE AD AS A FUNCTION OF GRAMMATICAL GENDER, PARTICIPANT GENDER, AND PRIMING CONDITION (STUDY 6) 

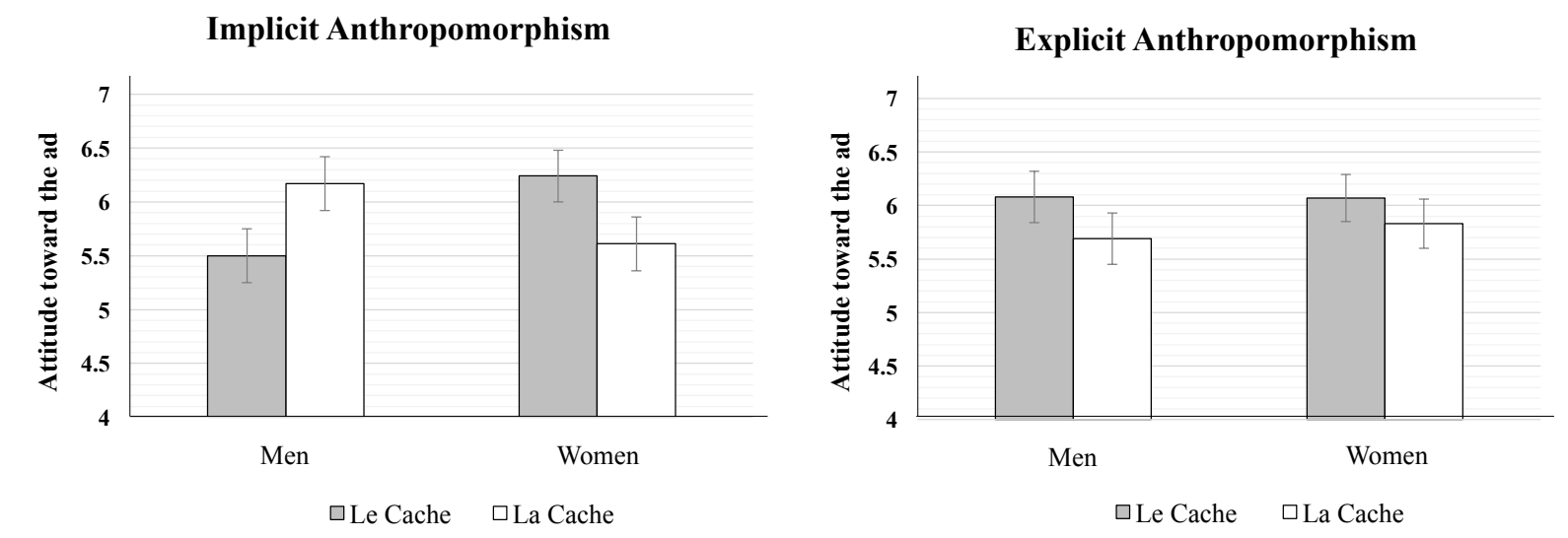

GENERAL DISCUSSION

The concept of anthropomorphism has been around for centuries. It is now considered a natural human tendency that everyone displays to some degree. However, this tendency to spontaneously attribute human characteristics to non-human entities varies across individuals, cultures, and situations. In the present research, we questioned what makes some consumers more likely to anthropomorphize than others. We identified language as a novel antecedent of anthropomorphism, and in particular, a language's grammatical gender system. Some languages, such as English, grammatically distinguish non-human entities through pronouns (it), whereas other languages, lack a separate pronoun for non-humans (it-less languages), and instead either 
gender-mark all objects, whether human or non-human (e.g., French) or do not gender-mark any objects, either human or non-human (e.g., Turkish).

Across 10 studies, we provide extensive evidence that these grammatical distinctions matter: speakers of it-less languages are more prone to anthropomorphism than are speakers of non-it-less languages (in particular, English). In the first six studies, we demonstrate that this effect is robust across different it-less languages (French, Turkish) and different operationalizations of anthropomorphism (trait measures, categorization tasks, text analysis of pronoun usage in discourse). We also provide causal evidence of the effect by manipulating pronoun usage through a learning task. In the remaining four studies, we provide evidence of these language-induced effects on anthropomorphism on consumer judgments. We show that speakers of an it-less language (French) rely on linguistic features such as the grammatical gender to characterize the anthropomorphized entity (the product/brand), even though the assignment of gender markers to non-human entities is semantically arbitrary. When participants were in effect asked to form an impression of a brand, attitudes were more favorable and willingness to pay was greater when the grammatical gender of the product was congruent with the gender of the participant, and the effect also held for consequential choice. However, when participants anticipated interacting with the brand, the congruence effect reversed, and attitudes were more favorable when the grammatical gender was opposite of participant gender.

Linguistic Relativity: Language Shapes Thought

At the most general level, our research contributes to literature on the effects of language on thought, providing support for the Sapir-Whorf hypothesis of linguistic relativity. Our 
research shows that particular language structures, such as grammatical gender, influence cognition. These results confirm and extend the previous findings on the effect of grammatical gender on object descriptions (Boroditsky, Schmidt, and Phillips 2003) and categorization (Yorkston and De Mello 2005). Furthermore, consistent with conceptualizations of linguistic relativity, our findings suggest that the effects are automatic, and occur outside of conscious awareness. Grammatical gender markings for non-human entities in it-less languages are semantically arbitrary, and speakers of it-less languages do not consciously attribute gender characteristics to these non-human entities. Thus, as we showed in study 6 , judgments of femininity/masculinity of a gender-marked brand were virtually identical for the feminine and masculine gender marks. However, despite any apparent conscious attribution of the gender marks to characteristics of the brand, the grammatical gender marks influenced higher-level cognitive mechanisms such as consumer attitudes, intentions, and choice.

Our research also contributes to the literature on psycholinguistic effects on informationprocessing and consumer judgments. A number of linguistic devices have been identified that shape consumer perceptions and judgments (Pogacar, Shrum, and Lowrey 2018). Grammatical gender marking represents an additional device. Thus, just as research on phonetic symbolism research shows the mere sound of a brand name can influence downstream consumer perceptions, attitudes, and behaviors, so too can the arbitrary gender marking of brand names.

Antecedents of Anthropomorphism

Our research also provides insights into the determinants of anthropomorphism. Epley et al. (2007) proposed a three-factor model of antecedents of anthropomorphism. Two are 
motivational (social connection, effectance), and one is cognitive (elicited agent knowledge). Elicited agent knowledge refers to the accessibility of knowledge structures, and our research is related to this factor. Our findings suggest that grammatical gender systems influence the accessibility and content of knowledge structures when reasoning about non-human entities. A language such as English, which grammatically distinguishes between humans and non-humans, highlights differences between humans and non-humans, putting them in different categories. Thus, differences (rather than similarities) between humans and non-humans are chronically accessible through language usage, which we suggest results in reduced tendencies to anthropomorphize non-human entities. In contrast, it-less languages such as French and Turkish do not make this distinction, and thus differences are likely to be less accessible, resulting in greater anthropomorphic tendencies compared to non-it-less languages.

Our findings raise a crucial issue concerning the current conceptualizations of anthropomorphism that equate anthropomorphism with a bias or an overgeneralized error (Guthrie 1993; Epley et al. 2008). These conceptualizations unknowingly discount the possibility of an alternative worldview in which people have chronically active human knowledge structures when reasoning about non-humans. In such a worldview, anthropomorphism can be approximated to the default mode of thinking, rather than an overgeneralized error, and may hold idiosyncratic consequences for individuals, as we show in this research. Thus, our research not only identifies a novel antecedent of anthropomorphism, but also scrutinizes the conceptual foundations of anthropomorphism research: it is possible that these foundations are artifacts of a linguistic ideology that is propogated by the dominant scientific language in psychology (i.e., English). 
Consumer Anthropomorphism

Our research also contributes to the literature on consumer anthropomorphism. To date, most research on consumer anthropomorphism has focused on the effects of anthropomorphism of brands and products on consumer judgments, but relatively little research has investigated the antecedents of anthropomorphism in consumer contexts (Yang et al. 2018). Because anthropomorphism can transform the nature of consumers' relations with brands and products, we argue that understanding what factors make some consumers more likely to anthropomorphize than other consumers, and whether these factors have idiosyncratic consequences on the way consumers anthropomorphize products and brands, is an important question.

Our findings indicate that grammatical gender of brand names and products influence consumer judgments, at least for speakers of it-less languages, even though the gender markings have no informational value. Merely changing the gender marks (masculine vs. feminine) influenced consumer judgments, and did so in ways consistent with how humans interact with other humans. For example, people generally like people who share similar personal characteristics as themselves (Byrne 1971; Turner 1985) and this in-group bias has been shown for human-non-human interactions as well (e.g., with robots; Eyssel et al. 2012). We also demonstrate this in-group bias as a function of grammatical gender-markings. Products are evaluated more favorably when grammatical gender and participant gender are congruent. However, in personal interactions, people often prefer to interact with someone of the opposite sex (Hall 1993; Moshavi 2004), and this preference has also been shown to apply to human-nonhuman (robot) interactions (Park et al. 2011; Siegel et al. 2009). Consequently, as we show, the 
gender congruence effect reverses when consumers anticipate an interaction with the product or brand. This pattern of effects suggest that speakers of it-less languages exhibit a type of grammatical anthropomorphism, nonconsciously using grammatical gender in forming their judgments about products.

Our findings have additional implications for consumer research on anthropomorphism, particularly in terms of the underlying processes. One pertains to how anthropomorphism is activated. For example, most consumer research primes anthropomorphism through explicit cues of a humanlike form (e.g., appearance, voice, and behavioral characteristics). Our research shows that priming the humanlike form is not essential for people to anthropomorphize consumption objects. Speakers of languages whose grammatical structure blurs the distinction between human-non-human categories already attribute human characteristics to consumption objects without any additional cue in the environment. In fact, our research shows that for grammatical anthropomorphism, the explicit cues are counterproductive for inducing anthropomorphism effects. Explicitly priming anthropomorphism (study 6) actually eliminates the grammatically induced anthropomorphism because it calls attention to the source of the bias, which consumers then correct for.

Limitations and Future Research

Our research provides an initial test of the proposition that grammatical gender systems influence anthropomorphism. We specifically chose to compare English and French speakers in most studies for several reasons, some practical. English and French are ubiquitous languages (first and tenth most commonly spoken; Dorren 2018), and thus are relevant to a large number of 
consumers. Second, in terms of maximizing internal validity, we wanted to avoid issues involved with multiple translations. There were also issues of our own access to participants and language expertise. Thus, a more comprehensive study across multiple languages would be beneficial. That said, recent research investigating the effects of grammatical gender systems on gender prejudice (DeFranza et al. 2020) and gender equality (Prewitt-Freilino, Caswell, and Laakso 2012) compared across multiple countries (45 and 101, respectively). Thus, the generalizability of the effect, at least in terms of gender equality and prejudice, is firmly established.

We did include one study comparing native Turkish and English speakers. Although the generalizability is still limited, the findings highlight an important issue for the processes underlying these effects. Turkish and French are both it-less languages, and as predicted, they produced similar effects relative to English. However, the grammatical gender systems of French and Turkish are also quite different, and this difference points toward possible process explanations. French is a gendered language; it does not grammatically distinguish between humans and non-humans, but instead assigns grammatical gender marks to all non-human objects. Consequently, it is impossible to determine whether the grammatical anthropomorphism occurs because of the absence of $i t$, or the presence of gender-marking for non-human objects (or both). In contrast, Turkish is also an it-less language, but does not assign gender to any object. Thus, for the Turkish language findings, the results indicate that it is the absence of the separate pronoun for non-human entities, and not the presence of gendered markings. Future research would benefit from further teasing apart the competing possibilities.

The conceptualization of reality corresponds in large part to one's language. Our research shows that one's natural tendency to anthropomorphize largely depends on whether the language's grammar system makes a distinction between humans and non-humans. For speakers 
of languages that do have this grammatical distinction (e.g., English), their subjective reality is built on a divide between humans and non-humans, creating a worldview that turns anthropomorphism into a transgression. Studying anthropomorphism across linguistic borders allows us to look at the otherwise-inaccessible angles of the phenomenon, which underlines the importance of psycholinguistics in studying consumer behavior and encourages researchers to find the linguistic walls inside which subjective realities shape the human mind. 


\section{REFERENCES}

Aggarwal, Pankaj and Ann L. McGill (2007), "Is That Car Smiling at Me? Schema Congruity as a Basis for Evaluating Anthropomorphized Products," Journal of Consumer Research, 34 (4), 468-79.

Ahn, Hee-Kyung, Hae Joo Kim, and Pankaj Aggarwal (2014), "Helping Fellow Beings: Anthropomorphized Social Causes and the Role of Anticipatory Guilt," Psychological Science, 25 (1), 224-29.

Alba, Joseph W. and J. Wesley Hutchinson (1987), "Dimensions of Consumer Expertise," Journal of Consumer Research, 13 (4), 411-54.

Alexander, Hartley Burr (1916), The Mythology of All Races - Volume X, Boston: The Archaeological Institute of America, Marshall Jones Company.

Anggoro, Florencia K., Sandra R. Waxman, and Douglas L. Medin (2008), "Naming Practices and the Acquisition of Key Biological Concepts: Evidence from English and Indonesian," Psychological Science, 19 (4), 314-19.

Bargh, John A. (1992), "Why Subliminality Does Not Matter to Social Psychology: Awareness of the Stimulus Versus Awareness of Its Influence," in Perception without Awareness, ed. Robert F. Bornstein \& Thane S. Pittman, New York: Guilford, 236-55.

Boroditsky, Lera, Lauren A. Schmidt, and Webb Phillips (2003), "Sex, Syntax, and Semantics," in Language in Mind: Advances in the Study of Language and Thought, ed. Dedre Gentner and Susan Goldin-Meadow, Cambridge, MA: The MIT Press, 61-79.

Boutonnet, Bastien, Panos Athanasopoulos, and Guillaume Thierry (2012), "Unconscious Effects of Grammatical Gender during Object Categorisation," Brain Research, 1479, 72-79.

Byrne, Donn Erwin (1971), The Attraction Paradigm, New York: Academic Press. 
Casasanto, Daniel (2016), "Linguistic Relativity," in The Routledge Handbook of Semantics, ed. Nick Reimer, New York: Routledge, 158-74.

Casasanto, Daniel and Lera Boroditsky (2008), "Time in the Mind: Using Space to Think About Time," Cognition, 106 (2), 579-93.

Chandler, Jesse and Norbert Schwarz (2010), "Use Does Not Wear Ragged the Fabric of Friendship: Thinking of Objects as Alive Makes People Less Willing to Replace Them," Journal of Consumer Psychology, 20 (2), 138-45.

Chen, Fangyuan, Jaideep Sengupta, and Rashmi Adaval (2018), "Does Endowing a Product with Life Make one Feel More Alive? The Effect of Product Anthropomorphism on Consumer Vitality," Journal of the Association for Consumer Research, 3 (4), 503-13.

Chen, M. Keith (2013), "The Effect of Language on Economic Behavior: Evidence from Savings Rates, Health Behaviors, and Retirement Assets," The American Economic Review, 103 (2), 690-731.

Chen, Rocky Peng, Echo Wen Wan, and Eric Levy (2017), "The Effect of Social Exclusion on Consumer Preference for Anthropomorphized Brands," Journal of Consumer Psychology, 27 (1), 23-34.

Chen, Sylvia Xiaohua and Michael Harris Bond (2010), "Two Languages, Two Personalities? Examining Language Effects on the Expression of Personality in a Bilingual Context," Personality and Social Psychology Bulletin, 36 (11), 1514-28.

Cohen, Joel B. and Kunal Basu (1987), "Alternative Models of Categorization: Toward a Contingent Processing Framework," Journal of Consumer Research, 13 (4), 455-72. Dacey, Mike (2017), "Anthropomorphism as Cognitive Bias," Philosophy of Science, 84 (5), $1152-64$. 
DeFranza, David, Himanshu Mishra, and Arul Mishra (2020), "How Language Shapes Prejudice Against Women: An Examination Across 45 World Languages," Journal of Personality and Social Psychology, Advance online publication. https://doi.org/10.1037/pspa0000188.

Dorren, Gaston (2018), Babel: Around the World in Twenty Languages, New York: Grove Press. Epley, Nicholas, Scott Akalis, Adam Waytz, and John T. Cacioppo (2008), "Creating Social Connection Through Inferential Reproduction," Psychological Science, 19 (2), 114-20.

Epley, Nicholas, Adam Waytz, and John T. Cacioppo (2007), "On Seeing Human: A ThreeFactor Theory of Anthropomorphism," Psychological Review, 114 (4), 864-86.

Epp, Amber M. and Linda L. Price (2009) "The Storied Life of Singularized Objects: Forces of Agency and Network Transformation," Journal of Consumer Research, 36 (5), 820-37.

Eyssel, Friederike, Dieta Kuchenbrandt, Simon Bobinger, Laura de Ruiter, and Frank Hegel (2012), "'If You Sound Like Me, You Must Be More Human': On the Interplay of Robot and User Features on Human- Robot Acceptance and Anthropomorphism," 7th ACM/IEEE International Conference on Human-Robot Interaction, IEEE, 125-26.

Fussell, Susan R., Sara Kiesler, Leslie D. Setlock, and Victoria Yew (2008), "How People Anthropomorphize Robots," ACM/IEEE International Conference on Human-Robot Interaction, HRI, 145-52.

Gendron, Maria, Kristen A. Lindquist, Lawrance Barsalou, and Lisa Feldman Barrett (2012), "Emotion Words Shape Emotion Percepts," Emotion, 12 (2), 314-25.

Guthrie, Stewart Elliott (1993), Faces in the Clouds: A New Theory of Religion, Oxford: Oxford University Press. 
(2014), "Religion as Anthropomorphism at Çatalhöyük," in Religion at Work in a

Neolithic Society: Vital Matters, ed. Ian Hodder, New York: Cambridge University Press, 86-108.

Haley, Kevin J. and Daniel MT Fessler (2005), "Nobody's Watching?: Subtle Cues Affect Generosity in an Anonymous Economic Game," Evolution and Human Behavior, 26 (3), 245-56.

Hall, Elaine J. (1993)"Smiling, Deferring, and Flirting: Doing Gender by Giving 'Good Service'," Work and Occupations, (20) 4, 452-71.

Hanks, William F. (1990), Referential Practice: Language and Lived Space among the Maya, Chicago: University of Chicago Press.

Harris, Lasana T. and Susan T. Fiske (2008), "The Brooms in Fantasia: Neural Correlates of Anthropomorphizing Objects," Social Cognition, 26 (2), 210-23.

Heberlein, Andrea S. and Ralph Adolphs (2004), "Impaired Spontaneous Anthropomorphizing Despite Intact Perception and Social Knowledge," PNAS, 101 (19), 7487-91.

Heider, Fritz and Marianne Simmel (1944), "An Experimental Study of Apparent Behavior," The American Journal of Psychology, 57 (2), 243-59.

Hume, David (1957), The Natural History of Religion, Stanford, CA: Stanford University Press.

Ji, Li-Jun, Zhiyong Zhang, and Richard E. Nisbett (2004), "Is it Culture or is it Language? Examination of Language Effects in Cross-cultural Research on Categorization," Journal of Personality and Social Psychology, 87 (1), 57-65.

Jipson, Jennifer L. and Susan A. Gelman (2007), "Robots and Rodents: Children's Inferences about Living and Nonliving Kinds," Child Development, 78 (6), 1675-88. 
Kashima, Emiko S. and Yoshihisa Kashima (1998), "Culture and Language: The Case of Cultural Dimensions and Personal Pronoun Use," Journal of Cross-Cultural Psychology, $29(3), 461-86$.

Kennedy, John S. (1992), The New Anthropomorphism, Cambridge: Cambridge University Press.

Kim, Sara and Ann L. McGill (2011), "Gaming with Mr. Slot or Gaming the Slot Machine? Power, Anthropomorphism, and Risk Perception," Journal of Consumer Research, 38 (1), 94-107.

Labroo, Aparna A., Ravi Dhar, and Norbert Schwarz (2008), "Of Frog Wines and Frowning Watches: Semantic Priming, Perceptual Fluency, and Brand Evaluation," Journal of Consumer Research, 34 (6), 819-31.

Langacker, Ronald W. (1987), Foundations of Cognitive Grammar: Theoretical Prerequisites, Palo Alto: Stanford University Press.

Logan, Robert K. (1986), The Alphabet Effect: The Impact of the Phonetic Alphabet on the Development of Western Civilization, New York: William Morrow and Co.

Lombardi, Wendy J., E. Tory Higgins, and John A. Bargh (1987), "The Role of Consciousness in Priming Effects on Categorization: Assimilation versus Contrast as a Function of Awareness of the Priming Task," Personality and Social Psychology Bulletin, 13 (3), 411-29.

Lowrey, Tina M. and L. J. Shrum (2007), "Phonetic Symbolism and Brand Name Preference," Journal of Consumer Research, 34 (3), 406-14.

Lucy, John A. (1997), "Linguistic Relativity," Annual Review of Anthropology, 26 (1), 291-312. 
Luna, David, Torsten Ringberg, and Laura A. Peracchio (2008), "One Individual, Two Identities: Frame Switching among Biculturals," Journal of Consumer Research, 35 (2), 279-93. MacIntyre, Peter D. and Robert C. Gardner (1994), "The Subtle Effects of Language Anxiety on Cognitive Processing in the Second Language," Language Learning, 44 (2), 283-305.

Meteyard, Lotte, Bahador Bahrami, and Gabriella Vigliocco (2007), "Motion Detection and Motion Verbs: Language Affects Low-level Visual Perception," Psychological Science, $18(11), 1007-13$.

Moshavi, Dan (2004), "He Said, She Said: Gender Bias and Customer Satisfaction with PhoneBased Service Encounters," Journal of Applied Social Psychology, (34) 1, 162-76.

Mourey, James A., Jenny G. Olson, and Carolyn Yoon (2017), "Products as Pals: Engaging with Anthropomorphic Products Mitigates the Effects of Social Exclusion," Journal of Consumer Research, 44 (2), 1-18.

Mühlhäusler, Peter and Rom Harré (1990), Pronouns and People: The Linguistic Construction of Social and Personal Identity, Oxford: Blackwell.

Park, Eunil, Ki Joon Kim, and Angel P. Del Pobil (2011), "The Effects of a Robot Instructor's Positive vs. Negative Feedbacks on Attraction and Acceptance towards the Robot in Classroom," in International Conference on Social Robotics, Berlin, Heidelberg: Springer, 135-41.

Pinker, Steven (1994), The Language Instinct, New York: William Morrow \& Co.

Pogacar, Ruth, L. J. Shrum, and Tina M. Lowrey (2018), "The Effects of Linguistic Devices on Consumer Information Processing and Persuasion: A Language Complexity $\times$ Processing Mode Framework,” Journal of Consumer Psychology, 28 (4), 689-711. 
Prewitt-Freilino, Jennifer L., T. Andrew Caswell, and Emmi K. Laakso (2012), "The Gendering of Language: A Comparison of Gender Equality in Countries with Gendered, Natural Gender, and Genderless Languages," Sex Roles, 66 (3-4), 268-81.

Roberson, Debi, Jules Davidoff, Ian R.L. Davies, and Laura R. Shapiro (2005), "Color Categories: Evidence for the Cultural Relativity Hypothesis," Cognitive Psychology, 50 (4), 378-411.

Roberson, Debi, Hyensou Pak, and J. Richard Hanley (2008), "Categorical Perception of Colour in the Left and Right Visual Field is Verbally Mediated: Evidence from Korean," Cognition, 107 (2), 752-62.

Ross, Norbert, Douglas Medin, John D. Coley, and Scott Atran (2003), "Cultural and Experiential Differences in the Development of Folkbiological Induction," Cognitive Development, 18 (1), 25-47.

Scheelea, Dirk, Christine Schweringa, Jed T. Elison, Robert Spunt, Wolfgang Maier, and René Hurlemann (2015), "A Human Tendency to Anthropomorphize is Enhanced by Oxytocin," European Neuropsychopharmacoogy, 25 (10), 1817-23.

Siegel, Mikey, Cynthia Breazeal, and Michael I. Norton (2009), "Persuasive Robotics: The Influence of Robot Gender on Human Behavior," IEEE/RSJ International Conference on Intelligent Robots and Systems, 2563-67.

Sujan, Mita and Christine Dekleva (1987), "Product Categorization and Inference Making: Some Implications for Comparative Advertising," Journal of Consumer Research, 14 (3), $372-$ 78. 
Tam, Kim-Pong, Sau-Lai Lee, and Melody Manchi Chao (2013), "Saving Mr. Nature:

Anthropomorphism Enhances Connectedness to and Protectiveness toward Nature," Journal of Experimental Social Psychology, 49 (3), 514-21.

Tausczik, Yla R. and James W. Pennebaker (2010), "The Psychological Meaning of Words: LIWC and Computerized Text Analysis Methods," Journal of Language and Social Psychology, 29 (1), 24-54.

Teeuwen, Mark and Bernhard Scheid (2002), "Tracing Shinto in the History of Kami Worship." Japanese Journal of Religious Studies, 29 (3-4), 195-207.

Turner, John C. (1985), "Social Categorization and the Self-Concept: A Social Cognitive Theory of Group Behavior," Advances in Group Processes: Theory and Research, 2, 77-122

Von Humboldt, Wilhelm and Wilhelm Freiherr von Humboldt (1999), Humboldt: 'On Language': On the Diversity of Human Language Construction and Its Influence on the Mental Development of the Human Species, Cambridge: Cambridge University Press.

Waytz, Adam, John Cacioppo, and Nicholas Epley (2010), "Who Sees Human? The Stability and Importance of Individual Differences in Anthropomorphism," Perspectives on Psychological Science, 5 (3), 219-32.

Waytz, Adam, Joy Heafner, and Nicholas Epley (2014), "The Mind in the Machine: Anthropomorphism Increases Trust in an Autonomous Vehicle," Journal of Experimental Social Psychology, 52, 113-17.

Waytz, Adam, Carey K. Morewedge, Nicholas Epley, George Monteleone, Jia-Hong Gao, and John T. Cacioppo (2010), "Making Sense by Making Sentient: Effectance Motivation Increases Anthropomorphism," Journal of Personality and Social Psychology, 99 (3), 410-35. 
Whorf, Benjamin Lee (1952), "Language, Mind, and Reality," A Review of General Semantics, 9 (3), 167-88.

Woodward, Amanda L. (1999), "Infants' Ability to Distinguish between Purposeful and Nonpurposeful Behaviors," Infant Behavior and Development, 22 (2), 145-60.

Yang, Linyun W., Pankaj Aggarwal, and Ann L. McGill (2020), "The 3 C's of Anthropomorphism: Connection, Comprehension, and Competition," Consumer Psychology Review, 3 (1), 3-19.

Yorkston, Eric and Gustavo E. De Mello (2005), "Linguistic Gender Marking and Categorization," Journal of Consumer Research, 32 (2), 224-34. 